\title{
Scalar geometry and masses in Calabi-Yau string models
}

\author{
Daniel Farquet and Claudio A. Scrucca \\ Institut de Théorie des Phénomènes Physiques \\ Ecole Polytechnique Fédérale de Lausanne \\ CH-1015 Lausanne, Switzerland
}

\begin{abstract}
We study the geometry of the scalar manifolds emerging in the no-scale sector of Kähler moduli and matter fields in generic Calabi-Yau string compactifications, and describe its implications on scalar masses. We consider both heterotic and orientifold models and compare their characteristics. We start from a general formula for the Kähler potential as a function of the topological compactification data and study the structure of the curvature tensor. We then determine the conditions for the space to be symmetric and show that whenever this is the case the heterotic and the orientifold models give the same scalar manifold. We finally study the structure of scalar masses in this type of geometries, assuming that a generic superpotential triggers spontaneous supersymmetry breaking. We show in particular that their behavior crucially depends on the parameters controlling the departure of the geometry from the coset situation. We first investigate the average sGoldstino mass in the hidden sector and its sign, and study the implications on vacuum metastability and the mass of the lightest scalar. We next examine the soft scalar masses in the visible sector and their flavor structure, and study the possibility of realizing a mild form of sequestering relying on a global symmetry.
\end{abstract}




\section{Introduction}

The low-energy effective action of string models with minimal supersymmetry, obtained by compactification on a suitable internal manifold with an appropriate gauge bundle over it, displays a number of generic features in the weak-coupling and large-volume regime. One of these properties is that there are always at least two neutral chiral multiplets that universally appear. These are the dilaton $S$, which is related to the string coupling, and the overall Kähler modulus $T$, which is related to the volume of the compactification manifold. Besides these, one of course requires the presence of some non-vanishing number $n$ of charged chiral multiplets $\Phi^{\alpha}$, for the model to possibly be viable at the phenomenological level. Ignoring any other field, the structure of the low-energy effective theory for these fields is then quite universal and fixed [1]. The effective Kähler potential, which controls the kinetic energy, is dominated by a non-trivial classical contribution. Additional effects coming from possible classical background fluxes or quantum corrections can usually be neglected, since they represent small corrections to the non-vanishing classical result. One then finds the following simple expression:

$$
K=-\log (S+\bar{S})-\log \left(T+\bar{T}-1 / 3 \Phi^{\alpha} \bar{\Phi}^{\alpha}\right)^{3} .
$$

In this situation, the scalar manifold is thus the product of two maximally symmetric coset spaces, with constant sectional curvatures given by $R=-2$ for the first factor and $R=-2 / 3$ for the second factor:

$$
\mathcal{M}=\frac{S U(1,1)}{U(1)} \times \frac{S U(1,1+n)}{U(1) \times S U(n)} .
$$

The effective superpotential, which controls the potential energy, has instead an essentially trivial classical contribution. Additional effects coming from possible classical background fluxes (see for instance $[2,3,4]$ and [5] for a review) or non-perturbative quantum corrections like gaugino condensation $[6,7]$ may thus play a crucial role. One can then imagine an essentially arbitrary and generic result:

$$
W=W\left(S, T, \Phi^{\alpha}\right) .
$$

This gives a simplified picture of the minimal situation that might be expected in string models, which has been extensively studied in the past.

In practice, however, generic string models involve many more neutral moduli and the situation correspondingly gets much more involved. In particular, while the dilaton stays on its own the overall Kähler modulus gets in general replaced by a number $1+m$ of Kähler moduli $T^{A}$, out of which 1 controls the overall volume of the compactification manifold and the other $m$ the sizes of its non-trivial cycles. In addition, there may also be other qualitatively different types of non-minimal moduli, like for instance complex structure or bundle moduli. But these are not universally present and do not necessarily mix to the universal ones or the matter fields. It makes thus some sense to completely ignore these in a first attempt of generalization. In such a more general situation, the low-energy effective theory becomes less restricted and displays a much larger spectrum of possibilities [8]. The Kähler potential is still dominated by a classical contribution, but this is no longer completely fixed and rather depends on the topological data of the 
compactification manifold and the gauge bundle over it. It turns out that it takes the following general form, characterized by some real functions $N^{A}$ which are in principle arbitrary but usually quadratic, and some real function $Y$ which should be homogeneous of degree three in its arguments but not necessarily polynomial:

$$
K=-\log (S+\bar{S})-\log Y\left(T^{A}+\bar{T}^{A}-N^{A}\left(\Phi^{\alpha}, \bar{\Phi}^{\alpha}\right)\right) .
$$

In this situation, the scalar manifold is no longer completely fixed. More precisely, it is the product of a first factor which is the same maximally symmetric coset manifold as before, with constant sectional curvature $R=-2$, and a second factor which is now a more general space of the no-scale type, with sectional curvature $R=R_{Y, N}$ that is in general non-constant but still quite restricted:

$$
\mathcal{M}=\frac{S U(1,1)}{U(1)} \times \mathcal{M}_{Y, N}
$$

The superpotential is instead, as before, dominated by additional unknown effects and can thus a priori take an arbitrary and generic form:

$$
W=W\left(S, T^{A}, \Phi^{\alpha}\right)
$$

This gives a somewhat more realistic picture of the generic situation that might be expected in string models, which has been less studied so far.

One of the biggest phenomenological concerns in string models is spontaneous supersymmetry breaking. A first crucial issue is of course to understand the dynamical origin of this breaking within the microscopic theory. We shall however not touch this problem and simply assume very optimistically that while the Kähler potential $K$ is approximately fixed the effective superpotential $W$ can be completely generic. This can then lead to a vacuum that spontaneously breaks supersymmetry, with arbitrary values for the auxiliary fields of chiral multiplets. For simplicity, we shall assume that the values of the auxiliary fields of vector multiplets are negligible, as it naturally tends to be the case, and thus ignore vector multiplets altogether. A second important issue is then the structure of the scalar and fermion masses. For generic directions in the chiral multiplet space, these two kinds of masses significantly depend both on the form of $K$ and $W$, and therefore nothing very predictive can be said from the knowledge of the one without the other. It however turns out that while their common supersymmetric part depends more on $W$ than $K$, their relative non-supersymmetric splitting depends more on $K$ than $W$. This suggests that one may be able to derive some constraints on the structure of these splitting from the knowledge of $K$ without $W$. This is indeed the case, as can be seen from the fact that the general expression for this mass splitting depends only on the sigma-model Riemann tensor, which characterizes the geometry of the scalar manifold, and the Goldstino vector defined by the auxiliary fields, which characterizes the direction of supersymmetry breaking in field space. Assuming vanishing and stationary vacuum energy, one finds:

$$
\Delta m_{I \bar{J}}^{2}=-\left[R_{I \bar{J} K \bar{L}}-\frac{1}{3}\left(g_{I \bar{J}} g_{K \bar{L}}+g_{I \bar{L}} g_{K \bar{J}}\right)\right] F^{K} \bar{F}^{\bar{L}} .
$$

For a given $K$ the forms of $g_{I \bar{J}}$ and $R_{I \bar{J} K \bar{L}}$ are fixed, and by varying $W$ one may only change the point at which these are evaluated and the vector $F^{K}$ with which they are contracted. 
The vanishing of the cosmological constant moreover implies that $g_{K \bar{L}} F^{K} \bar{F}^{\bar{L}}=3 m_{3 / 2}^{2}$, and for fixed gravitino mass $m_{3 / 2}$ one may thus vary only the direction defined by the Goldstino unit vector $f^{I}=F^{I} /|F|$. The final observation is now that for those special directions in the chiral multiplet space for which supersymmetric mass terms are not allowed and vanish, any information on the non-supersymmetric splitting naturally translates into an information on the absolute masses too.

It turns out that there are two particularly important kinds of special directions of the above type in the chiral multiplet space, for which not only supersymmetric mass terms are not allowed, but the masses of the corresponding fermions are actually fixed while the masses for the corresponding scalars are entirely controlled by the above splitting effect. The first special direction is the Goldstino direction $f^{I}$ within the hidden subsector of fields with non-vanishing VEVs $[9,10]$. Indeed, in this direction the fermion is the would-be Goldstino mode, which would be forced to have a vanishing mass by the superGoldstone theorem in the rigid limit and is actually eaten by the gravitino through the superHiggs mechanism in the local case. As a result, the average mass of the two real sGoldstino scalar partners is equal to the splitting matrix along this direction, and thus reads

$$
m_{f \bar{f}}^{2}=\left(2-3 R_{I \bar{J} K \bar{L}} f^{I} \bar{f}^{\bar{J}} f^{K} \bar{f} \bar{L}\right) m_{3 / 2}^{2} .
$$

The second kind of special directions are actually all the flavor directions $v^{I}$ within the visible subsector of fields with vanishing VEVs and standard model quantum numbers $[11,12]$. Indeed, in this subspace the fermions correspond to the ordinary quarks and leptons and are thus not allowed to have direct mass terms, because these are forbidden by the standard model gauge symmetries. As a consequence, the mass matrix of the scalars in this sector, which are the squarks and sleptons, is equal to the splitting matrix along this subspace, and is therefore given by

$$
m_{v \bar{v}}^{2}=\left(1-3 R_{I \bar{J} K \bar{L}} v^{I} \bar{v}^{\bar{J}} f^{K} \bar{f}^{\bar{L}}\right) m_{3 / 2}^{2} .
$$

It is evident from the structure of eqs. (1.8) and (1.9) that the knowledge of the curvature tensor $R_{I \bar{J} K \bar{L}}$ of the scalar manifold is a quite crucial information. Indeed, for $m_{f \bar{f}}^{2}$ to be positive one needs the sectional curvature $R(f)=-R_{I \bar{J} K \bar{L}} f^{I} \bar{f}^{\bar{J}} f^{K} \bar{f}^{\bar{L}}$ in the plane defined by the vector $f^{I}$ to be larger than the critical value $-2 / 3$, while for $m_{v \bar{v}}^{2}$ to be universal and positive one needs the bisectional curvature $R(v, f)=-R_{I \bar{J} K \bar{L}} v^{I} \bar{v}^{\bar{J}} f^{K} \bar{f} \bar{L}$ in the planes defined by the orthogonal vectors $v^{I}$ and $f^{I}$ to be independent of $v^{I}$ and larger than the critical value $-1 / 3$. For the dilaton sector alone, the situation is fixed and one finds a negative contribution to $m_{f \bar{f}}^{2}$ and a universal contribution to $m_{v \bar{v}}^{2}$. For the no-scale sector on its own, there are instead various possibilities. In the simplest case of maximally symmetric manifold, one finds a vanishing contribution to $m_{f \bar{f}}^{2}$ and a vanishing contribution to $m_{v \bar{v}}^{2}[14,15,16]$. In the more general case of non-maximally but still symmetric manifolds, it has been shown through an exhaustive study of all the possible cases that the contribution to $m_{f \bar{f}}^{2}$ is always negative or at best vanishing [17], while the contribution to $m_{v \bar{v}}^{2}$ is always allowed to vanish and thus be trivially universal $[18,19]$. In the most general case of non-symmetric manifolds one has instead a richer set of possibilities which were only partly studied so far, both for the contribution to $m_{f \bar{f}}^{2}[20]$ and the one to $m_{v \bar{v}}^{2}[21]$. But one can show that there always exists at least one special 
choice for $f^{I}$ such that $R(f)=-2 / 3$ and $R(v, f)=-1 / 3$ for any $v^{I}$, leading to vanishing contributions for both $m_{f \bar{f}}^{2}$ and $m_{v \bar{v}}^{2}$. Notice finally that if one allows the addition of an extra unspecified sector to uplift the cosmological constant along the lines of [4], the situation becomes more flexible and the impact on hidden-sector and visible sector scalar masses has been partly studied in [22, 23] and [24]. But here we will exclude this option and only consider those fields that are honestly described by the effective theory.

The aim of this paper is to study the general structure of the curvature for spaces of the general type (1.5), focusing on the second factor $\mathcal{M}_{Y, N}$. We will first do this in full generality for arbitrary functions $Y$ and $N^{A}$, and compute both the Riemann tensor and its covariant derivative in terms of derivatives of these functions, in order to be able to describe how the geometry changes when departing from the particular case of symmetric manifolds. We will then specialize to the case of generic weekly coupled string models based on compactifications on a Calabi-Yau manifold with a holomorphic vector bundle over it, where the function $Y$ can be parametrized in terms of the intersection numbers $d_{A B C}$ of the Calabi-Yau manifold while the functions $N^{A}$ can be parametrized in terms of some matrices $c_{\alpha \beta}^{A}$ related to the topology of the gauge bundle over it. We will study both heterotic and orientifold constructions, and show that these two classes of models produce scalar manifolds which are in some sense dual to each other and coincide whenever they are symmetric manifolds. We will then study the conditions that $d_{A B C}$ and $c_{\alpha \beta}^{A}$ have to satisfy for these manifolds to be symmetric, and we will identify two kinds of combinations of these parameters, denoted by $a_{A B C D}$ and $b_{\alpha \beta}^{A B}$, which control the departure of such geometries from each other and from the common special case of symmetric spaces. We will finally use these results to study the structure of the average sGoldstino mass (1.8) and the soft scalar masses (1.9) in such models, as functions of the numbers $d_{A B C}$ and $c_{\alpha \beta}^{A}$, and investigate the conditions under which these may respectively be positive and flavor universal, as required by phenomenological considerations. Special attention will be devoted to the role of the parameters $a_{A B C D}$ and $b_{\alpha \beta}^{A B}$ that control the deviation from the symmetric situation.

The rest of the paper is organized as follows. In section 2 we study the curvature of a generic no-scale manifold. In sections 3 and 4 we specialize to the cases of heterotic and orientifold models and work out more concretely the form of the curvature tensor and the conditions under which it is covariantly constant. In section 5 we summarize the general features, similarities and differences of the scalar geometries emerging from these two classes of models, and discuss the common case where they are coset spaces. In section 6 we apply these results to the study of the average sGoldstino mass and derive the conditions under which this can be positive and thus allow for a metastable vacuum. In section 7 we similarly apply the same results to the study of the visible soft scalar masses and discuss the conditions under which these can be flavor universal. Finally, in section 8 we summarize our results and state our conclusions.

\section{General no-scale manifolds}

The scalar manifolds describing the no-scale sector of Kähler moduli and matter fields of a string model enjoy, as already said, some general features that strongly constrain their geometry. The coordinates $Z^{i}$ split into two subsets $T^{A}$ and $\Phi^{\alpha}$, such that the Kähler 
potential takes the following general form:

$$
K=-\log Y\left(J^{A}\right), \quad J^{A}=T^{A}+\bar{T}^{A}-N^{A}\left(\Phi^{\alpha}, \bar{\Phi}^{\alpha}\right) .
$$

The function $Y$ has the general property of being homogeneous of degree three, that is:

$$
J^{A} \frac{\partial}{\partial J^{A}} Y=3 Y
$$

The functions $N^{A}$ may instead be arbitrary, the only crucial property of the variables $J^{A}$ being that:

$$
\frac{\partial}{\partial J^{A}}=\frac{\partial}{\partial T^{A}}=\frac{\partial}{\partial \bar{T}^{A}}
$$

This type of manifolds, called no-scale manifolds, enjoys a series of simple special properties, which have far reaching physical consequences. In terms of $K=-\log Y$, the homogeneity constraint (2.2) implies that $J^{A} \partial K / \partial J^{A}=-3$. But $K_{i}=\partial K / \partial J^{A} \partial J^{A} / \partial Z^{i}$, and in particular $K_{A}=\partial K / \partial J^{A}$, so that this homogeneity constraint can be rewritten in the following simple form:

$$
K_{A} J^{A}=-3 .
$$

Taking a derivative of this relation, it follows that $K_{i A} J^{A}+K_{i}=0$. Acting then on this with the inverse metric one deduces that

$$
K^{i}=-\delta_{A}^{i} J^{A}
$$

Form these two properties it finally follows that there is a no-scale structure, namely:

$$
K_{i} K^{i}=3 \text {. }
$$

\subsection{Geometry}

Let us now examine the impact of these properties on the geometry. To start with, it is convenient to write down what happens for a generic Kähler manifold where the Kähler potential is parametrized as $K=-\log Y$, with completely arbitrary $Y$. It is straightforward to show that $K_{i}=-Y_{i} / Y$ and $K^{i}=\left(1-Y_{p} Y^{-1 p \bar{q}} Y_{\bar{q}} / Y\right)^{-1} Y^{-1 i \bar{\jmath}} Y_{\bar{\jmath}}$, and that the metric and its inverse can be written as

$$
\begin{aligned}
g_{i \bar{\jmath}} & =-\frac{Y_{i \bar{\jmath}}}{Y}+\frac{Y_{i} Y_{\bar{\jmath}}}{Y^{2}}, \\
g^{i \bar{\jmath}} & =-Y Y^{-1 i \bar{\jmath}}-\left(1-\frac{Y_{p} Y^{-1 p \bar{q}} Y_{\bar{q}}}{Y}\right)^{-1} Y^{-1 i \bar{r}} Y_{\bar{r}} Y^{-1 \bar{\jmath} s} Y_{s} .
\end{aligned}
$$

The Christoffel symbols are instead given by:

$$
\Gamma_{i j \bar{k}}=-\frac{Y_{i j \bar{k}}}{Y}+\frac{Y_{i j} Y_{\bar{k}}}{Y^{2}}-\frac{g_{i \bar{k}} Y_{j}+g_{j \bar{k}} Y_{i}}{Y} .
$$


The Riemann tensor is then found to read:

$$
\begin{aligned}
R_{i \bar{\jmath} p \bar{q}}= & g_{i \bar{\jmath}} g_{p \bar{q}}+g_{i \bar{q}} g_{p \bar{\jmath}}-\frac{Y_{i \bar{\jmath} p \bar{q}}}{Y}-\frac{Y_{i p \bar{s}} Y^{\bar{s}} \bar{q}}{Y^{2}} \\
& +\left(1-\frac{Y_{p} Y^{-1 p \bar{q}} Y_{\bar{q}}}{Y}\right)^{-1} \frac{Y_{i p} Y_{\bar{\jmath} \bar{q}}}{Y^{2}}+\frac{Y_{i p} Y_{\bar{\jmath} r} Y^{r}}{Y^{3}}+\frac{Y_{\bar{\jmath} \bar{q}} Y_{i p \bar{s}} Y^{\bar{s}}}{Y^{3}} .
\end{aligned}
$$

Finally, one may try to compute the covariant derivative of the Riemann tensor, but this produces a quite complicated expression:

$$
\nabla_{n} R_{i \bar{\jmath} p \bar{q}}=\text { complicated expression } .
$$

Let us now see what happens if the manifold is of the no-scale type discussed above. The generic no-scale property $K_{i} K^{i}=3$ has a very simple and straightforward consequence. Indeed, it implies that $\left(Y_{i} Y^{-1 i \bar{\jmath}} Y_{\bar{\jmath}} / Y\right)\left(1-Y_{p} Y^{-1 p \bar{q}} Y_{\bar{q}} / Y\right)^{-1}=-3$, that is:

$$
Y_{i} Y^{i \bar{\jmath}} Y_{\bar{\jmath}}=\frac{3}{2} Y
$$

The more specific homogeneity properties $K_{A} J^{A}=-3$ and $K^{i}=-\delta_{A}^{i} J^{A}$, which also imply the no-scale property $K_{i} K^{i}=3$, have further consequences. They respectively imply that $Y_{A} J^{A}=3 Y$ and $Y^{i \bar{r}} Y_{\bar{r}}=1 / 2 \delta_{A}^{i} J^{A}$, which also give the no-scale property $Y_{i} Y^{i \bar{\jmath}} Y_{\bar{\jmath}}=3 / 2 Y$. To work out the most relevant implications of these relations, we may start from the noscale relation written as $Y_{l} K^{l}=-3 Y$, and deduce by taking further derivatives and making use of the homogeneity properties that $Y_{i l} K^{l}=-2 Y_{i}, Y_{i j l} K^{l}=-Y_{i j}, Y_{i j k l} K^{l}=0$ and $Y_{\bar{\imath} l} K^{l}=-2 Y_{\bar{\imath}}, Y_{\bar{\imath} \bar{l} l} K^{l}=-Y_{\bar{\imath} \bar{\jmath}}, Y_{\bar{\imath} \bar{k} l} K^{l}=0$. Finally, recalling that $K_{i}=-Y_{i} / Y$ and thus $K^{i}=-Y^{i} / Y$, one arrives at the following relations:

$$
\begin{aligned}
& Y_{l} Y^{l}=3 Y^{2}, \\
& Y_{i l} Y^{l}=2 Y Y_{i}, \quad Y_{\bar{l} l} Y^{l}=2 Y Y_{\bar{\imath}}, \\
& Y_{i j l} Y^{l}=Y Y_{i j}, \quad Y_{\bar{\imath} \bar{l} l} Y^{l}=Y Y_{\bar{\imath} \bar{\jmath}}, \\
& Y_{i j k l} Y^{l}=0, \quad Y_{\bar{\imath} \bar{\jmath} l} Y^{l}=0 .
\end{aligned}
$$

By taking various derivatives of these relations, one can also further deduce that:

$$
\begin{aligned}
& Y_{i \bar{\jmath} l} Y^{l}=-2 g_{i \bar{\jmath}}+Y_{i k} Y_{\bar{\jmath}}^{k} \\
& Y_{i j \bar{k} l} Y^{l}=Y Y_{i j \bar{k}}-Y_{i j} Y_{\bar{k}}+Y_{i j l} Y_{\bar{k}}^{l}, \\
& Y_{i j}{ }^{l} Y_{l k}=-Y Y_{i j k}+Y_{i j} Y_{k}, \\
& Y Y_{i \bar{\jmath} \bar{k} l} Y^{l}=Y^{2} Y_{i \bar{\jmath} \bar{k}}+Y Y_{i} Y_{\bar{\jmath} \bar{k}}-Y_{\bar{\jmath} k l} Y^{l m} Y_{m p} .
\end{aligned}
$$

Coming back to the geometry, we now see that the relation (2.12) descending from the no-scale property implies that the factor $\left(1-Y_{p} Y^{-1 p \bar{q}} Y_{\bar{q}} / Y\right)^{-1}$ appearing in (2.8) and (2.10) simplifies to a constant and is equal to -2 , while the properties (2.15) descending from homogeneity further imply that the last three terms of (2.10) cancel out. To sum up, one then has $K_{i}=-Y_{i} / Y$ and $K^{i}=-2 Y^{-1 i \bar{\jmath}} Y_{\bar{\jmath}}=-\delta_{A}^{i} J^{A}$, and the metric and its inverse read:

$$
\begin{aligned}
g_{i \bar{\jmath}} & =-\frac{Y_{i \bar{\jmath}}}{Y}+\frac{Y_{i} Y_{\bar{\jmath}}}{Y^{2}}, \\
g^{i \bar{\jmath}} & =-Y Y^{i \bar{\jmath}}+2 Y^{-1 i \bar{r}} Y_{\bar{r}} Y^{-1 \bar{\jmath} s} Y_{s} .
\end{aligned}
$$


The Christoffel symbols are as before

$$
\Gamma_{i j \bar{k}}=-\frac{Y_{i j \bar{k}}}{Y}+\frac{Y_{i j} Y_{\bar{k}}}{Y^{2}}-\frac{g_{i \bar{k}} Y_{j}+g_{j \bar{k}} Y_{i}}{Y} .
$$

The Riemann tensor is instead given by the following very simple expression:

$$
R_{i \bar{\jmath} p \bar{q}}=g_{i \bar{\jmath}} g_{p \bar{q}}+g_{i \bar{q}} g_{p \bar{\jmath}}-\frac{Y_{i \bar{\jmath} p \bar{q}}}{Y}-\frac{Y_{i p \bar{s}} Y_{\bar{\jmath} \bar{q}}^{\bar{\jmath}}}{Y^{2}}
$$

Finally, after a straightforward computation using several of the above identities, the covariant derivative of the curvature tensor is found to be completely symmetric in its holomorphic and antiholomorphic indices as implied by the Bianchi identity and reads

$$
\begin{aligned}
\nabla_{n} R_{i \bar{\jmath} p \bar{q}}= & g_{n \bar{\jmath}} \Gamma_{i p \bar{q}}+g_{i \bar{\jmath}} \Gamma_{n p \bar{q}}+g_{p \bar{\jmath}} \Gamma_{i n \bar{q}}+g_{n \bar{q}} \Gamma_{i p \bar{\jmath}}+g_{i \bar{q}} \Gamma_{n p \bar{\jmath}}+g_{p \bar{q}} \Gamma_{i n \bar{\jmath}} \\
& -\Gamma_{n i}{ }^{k} R_{k \bar{\jmath} p \bar{q}}-\Gamma_{n p}{ }^{k} R_{k \bar{\jmath} i \bar{q}}-\Gamma_{n p}{ }^{k} R_{k \bar{\jmath} i \bar{q}}-\frac{Y_{n} R_{i \bar{\jmath} p \bar{q}}+Y_{i} R_{n \bar{\jmath} p \bar{q}}+Y_{p} R_{i \bar{\jmath} n \bar{q}}}{Y} \\
& +\frac{Y_{n} g_{i \bar{\jmath}} g_{p \bar{q}}+Y_{i} g_{n \bar{\jmath}} g_{p \bar{q}}+Y_{p} g_{i \bar{\jmath}} g_{n \bar{q}}+Y_{n} g_{i \bar{q}} g_{p \bar{\jmath}}+Y_{i} g_{n \bar{q}} g_{p \bar{\jmath}}+Y_{p} g_{i \bar{q}} g_{n \bar{\jmath}}}{Y} \\
& -\frac{Y_{n i p \bar{q} \bar{\jmath}}}{Y}-\frac{Y_{n i p} Y_{\bar{q} \bar{\jmath}}}{Y^{2}}-\frac{Y_{n i p}{ }^{k} Y_{k \bar{q} \bar{\jmath}}}{Y^{2}} .
\end{aligned}
$$

The above expressions represent novel general results for the form of the geometry of no-scale manifolds, which are rather similar in form to those holding for special Kähler manifolds. We see that generic no-scale manifolds are neither homogeneous nor symmetric, since a priori $\nabla_{n} R_{i \bar{\jmath} p \bar{q}} \neq 0$. On the other hand, there exist particular no-scale manifolds which are coset spaces, and correspondingly $\nabla_{n} R_{i \bar{\jmath} p \bar{q}}=0$, but clearly there can be only a finite set of these, and this must be a subset of all the possible Kähler coset manifolds described in [25].

\subsection{General properties}

Using the various identities listed above, it is straightforward to show that all the geometrical quantities that have been computed enjoy some universal properties along the special direction defined by the unit vector

$$
k^{i}=-\frac{1}{\sqrt{3}} K^{i} .
$$

For the metric on has

$$
\begin{aligned}
& g_{i \bar{\jmath}} \bar{k}^{\bar{\jmath}}=\bar{k}_{i}, \\
& g_{i \bar{\jmath}} k^{i} \bar{k}^{\bar{\jmath}}=1,
\end{aligned}
$$

for the Christoffel symbols

$$
\begin{aligned}
& \Gamma_{i j \bar{k}} \bar{k}^{\bar{k}}=-\frac{2}{\sqrt{3}} K_{i j}, \quad \Gamma_{i j \bar{k}} k^{j}=-\frac{1}{\sqrt{3}}\left(g_{i \bar{k}}+K_{i l} K_{\bar{k}}^{l}\right), \\
& \Gamma_{i j \bar{k}} k^{j} \bar{k}^{\bar{k}}=-\frac{2}{\sqrt{3}} \bar{k}_{i}, \\
& \Gamma_{i j \bar{k}} k^{i} k^{j} \bar{k}^{\bar{k}}=-\frac{2}{\sqrt{3}},
\end{aligned}
$$


for the Riemann tensor

$$
\begin{aligned}
& R_{i \bar{\jmath} p \bar{q}} \bar{k}^{\bar{q}}=-\frac{1}{\sqrt{3}} \Gamma_{i p \bar{\jmath}}, \\
& R_{i \bar{\jmath} p \bar{q}} k^{p} \bar{k}^{\bar{q}}=\frac{1}{3}\left(g_{i \bar{\jmath}}+K_{i l} K_{\bar{\jmath}}^{l}\right), \quad R_{i \bar{\jmath} p \bar{q}} \bar{k}^{\bar{\jmath}} \bar{k}^{\bar{q}}=\frac{2}{3} K_{i p}, \\
& R_{i \bar{\jmath} p \bar{q}} \bar{k}^{\bar{\jmath}} k^{p} \bar{k}^{\bar{q}}=\frac{2}{3} \bar{k}_{i}, \\
& R_{i \bar{\jmath} p \bar{q}} k^{i} \bar{k}^{\bar{\jmath}} k^{p} \bar{k}^{\bar{q}}=\frac{2}{3},
\end{aligned}
$$

and finally for the covariant derivative of the Riemann tensor

$$
\begin{aligned}
& \nabla_{n} R_{i \bar{\jmath} p \bar{q}} k^{p}=0, \quad \nabla_{n} R_{i \bar{\jmath} p \bar{q}} \bar{k}^{\bar{q}}=-\frac{1}{\sqrt{3}}\left(\nabla_{n} \Gamma_{i \bar{\jmath} p}+R_{i \bar{\jmath} p \bar{q}} K_{s}^{\bar{q}}\right), \\
& \nabla_{n} R_{i \bar{\jmath} p \bar{q}} k^{i} k^{p}=0, \quad \nabla_{n} R_{i \bar{\jmath} p \bar{q}} k^{p} \bar{k}^{\bar{q}}=0, \quad \nabla_{n} R_{i \bar{\jmath} p \bar{q}} \bar{k}^{\bar{\jmath}} \bar{k}^{\bar{q}}=0, \\
& \quad \cdots \\
& \nabla_{n} R_{i \bar{\jmath} p \bar{q}} k^{n} k^{i} \bar{k}^{\bar{\jmath}} k^{p} \bar{k}^{\bar{q}}=0 .
\end{aligned}
$$

With the help of the above properties, it is now straightforward to demonstrate that the sectional curvature in the plane defined by the special vector $k^{i}$ is equal to $-2 / 3$ and that the bisectional curvature in the planes defined by the special vector $k^{i}$ and any orthogonal vector $v^{i}$ in the visible subsector is equal to $-1 / 3$, as anticipated in the introduction: $R(k)=-2 / 3$ and $R(v, k)=-1 / 3$.

\section{$3 \quad$ Heterotic models}

Let us now consider the more specific case of heterotic string models compactified on a Calabi-Yau manifold $X$ with a holomorphic vector bundle over it $[26,27,28]$. In this setting, the Kähler moduli $T^{A}$ are associated to harmonic $(1,1)$ forms $\omega_{A}$ on $X$, which are dual to the harmonic $(2,2)$ forms $\omega^{A}$ on $X$, while the matter fields $\Phi^{\alpha}$ are associated to bundle-valued harmonic $(1,0)$ forms $u_{\alpha}$ on $X$. The relevant numbers defining the low-energy effective theory are then given by the following integrals:

$$
\begin{aligned}
& d_{A B C}=\int_{X} \omega_{A} \wedge \omega_{B} \wedge \omega_{C}, \\
& c_{\alpha \beta}^{A}=\int_{X} \omega^{A} \wedge \operatorname{tr}\left[u_{\alpha} \wedge \bar{u}_{\beta}\right] .
\end{aligned}
$$

We will take the point of view that a priori $d_{A B C}$ can be an arbitrary symmetric symbol and similarly that $c_{\alpha \beta}^{A}$ can be an arbitrary set of Hermitian matrices, and study the lowenergy effective scalar geometry as a function of these parameters.

\subsection{Kähler potential}

The effective Kähler potential for the Kähler moduli $T^{A}$ and the matter fields $\Phi^{\alpha}$ can be worked out by dimensionally reducing the kinetic terms of the ten-dimensional supergravity theory describing the heterotic string below the Planck scale down to four dimensions,

retaining only the harmonic components of the fields. The full moduli dependence was 
worked out in $[29,30]$, and the leading matter field dependence in [31], while the full matter field dependence was studied only more recently in $[21,32]$ (see also [33, 34, 35]), generalizing the results that were available from [8] for the special case of orbifold limits. The complete result depends on the parameters $d_{A B C}$ and $c_{\alpha \beta}^{A}$ and takes the form $K=-\log Y$, where the function $Y$ depends only on certain combinations of fields. More precisely, we have

$$
Y=\mathcal{V}
$$

where $\mathcal{V}$ denotes the volume of the Calabi-Yau manifold and is given by the following expression in terms of the real geometric moduli fields $v^{A}$ :

$$
\mathcal{V}=\frac{1}{6} d_{A B C} v^{A} v^{B} v^{C} .
$$

The real fields $v^{A}$ are then linked to the following real combination of complex fields:

$$
J^{A}=T^{A}+\bar{T}^{A}-c_{\alpha \beta}^{A} \Phi^{\alpha} \bar{\Phi}^{\beta} .
$$

The relation between the $v^{A}$ and the $J^{A}$ is in this case trivial and given simply by

$$
J^{A}=v^{A} .
$$

It follows that $Y$ can be explicitly written in terms of the variables $J^{A}$ and simply reads

$$
Y=\frac{1}{6} d_{A B C} J^{A} J^{B} J^{C}
$$

We see that $Y$ is a homogenous function of degree three in the variables $J^{A}$, and we therefore have a no-scale manifold. Moreover, the function $Y$ is in this case a simple cubic polynomial in the variables $J^{A}$, involving only integer powers of them.

\subsection{Canonical parametrization}

We now want to study the above space at a given reference point, which can be thought of as the one defined by the $\operatorname{VEVs}\left\langle T^{A}\right\rangle$ and $\left\langle\Phi^{\alpha}\right\rangle$ that the scalar fields eventually acquire in the presence of a non-trivial superpotential. For simplicity, we shall restrict to the situation where the moduli have sizable VEVs whereas the matter fields have negligible VEVs, that is:

$$
\left\langle T^{A}\right\rangle \neq 0,\left\langle\Phi^{\alpha}\right\rangle=0 .
$$

For any given reference point of the type (3.8), it is possible to define a particularly convenient canonical parametrization, in such a way as to simplify the form of the metric, the Christoffel symbols and the curvature tensor at that point. To this aim, we proceed along the lines of $[36,37]$ and consider the field redefinitions

$$
\hat{T}^{A}=U_{B}^{A} T^{B}, \quad \hat{\Phi}^{\alpha}=V_{\beta}^{\alpha} \Phi^{\beta},
$$

together with the parameter redefinitions

$$
\hat{d}_{A B C}=\alpha U_{A}^{-1 D} U_{B}^{-1 E} U_{C}^{-1 F} d_{D E F}, \quad \hat{c}_{\alpha \beta}^{A}=U_{B}^{A} V_{\alpha}^{-1 \gamma} \bar{V}_{\beta}^{-1 \delta} c_{\gamma \delta}^{B} .
$$


Under the above combined transformations, with $U_{B}^{A}$ a real matrix, $V_{\beta}^{\alpha}$ a complex matrix and $\alpha$ a positive real number, the real geometrical moduli transform simply as $\hat{v}^{A}=U_{B}^{A} v^{B}$ and the Kähler potential remains unchanged, modulo an irrelevant Kähler transformation:

$$
\hat{K}=K-\log \alpha .
$$

We may now choose $U_{B}^{A}$ and $V_{\beta}^{\alpha}$ in such a way that the VEVs of the fields are aligned along just one direction, the VEV of the metric becomes diagonal, and the overall scale of these two quantities is set to some reference value. We may furthermore choose $\alpha$ to set the overall scale of the intersection numbers to a convenient value. More specifically, we shall require that in the new basis the reference point should be at

$$
\left\langle\hat{T}^{A}\right\rangle=\frac{\sqrt{3}}{2} \delta_{0}^{A}+i(\cdots),\left\langle\hat{\Phi}^{\alpha}\right\rangle=0,
$$

the metric at that point should take the form

$$
\left\langle\hat{g}_{A \bar{B}}\right\rangle=\delta_{A B},\left\langle\hat{g}_{\alpha \bar{\beta}}\right\rangle=\delta_{\alpha \beta},\left\langle\hat{g}_{A \bar{\beta}}\right\rangle=0,
$$

and finally the Kähler frame should be such that at that point

$$
\langle\hat{K}\rangle=0 \text {. }
$$

It is easy to get convinced by a counting of parameters that it is indeed always possible to impose this kind of conditions. Moreover, by comparing the expressions for the VEVs of the fields, the metric and the Kähler potential with the values required in the previous equations, we deduce that the new values of the numerical coefficients $\hat{d}_{A B C}$ and $\hat{c}_{\alpha \beta}^{A}$ must take the following form:

$$
\begin{aligned}
& \hat{d}_{000}=\frac{2}{\sqrt{3}}, \quad \hat{d}_{00 a}=0, \quad \hat{d}_{0 a b}=-\frac{1}{\sqrt{3}} \delta_{a b}, \quad \hat{d}_{a b c}=\text { generic }, \\
& \hat{c}_{\alpha \beta}^{0}=\frac{1}{\sqrt{3}} \delta_{\alpha \beta}, \quad \hat{c}_{\alpha \beta}^{a}=\text { generic } .
\end{aligned}
$$

Notice finally that from the point of view of the Calabi-Yau manifold, the canonical frame just corresponds to a convenient choice of basis for harmonic forms, which is suitably oriented with respect to the Kähler form and normalized in such a way as to get unit volume, since $\left\langle\hat{v}^{A}\right\rangle=\sqrt{3} \delta_{0}^{A}$ and $\langle\hat{\mathcal{V}}\rangle=1$. Moreover, by comparing with the general results of section 2 we see that the canonical frame essentially corresponds to choosing a parametrization such that the special direction $k^{i}$ is identified with one of the fields, since $\left\langle\hat{k}^{i}\right\rangle=\delta_{0}^{i}$.

\subsection{Geometry}

Let us now explore the geometry at a given reference point by using the new canonical coordinates. For notational simplicity, we drop from now on the hats referring to the definition of this special frame, and also the brackets referring to the special point.

We start by computing the first five partial derivatives of $\mathcal{V}$, which are the basic ingredients that we need. It is convenient to introduce the following notation:

$$
\begin{aligned}
& d_{A B}=d_{A B C} J^{C}, \\
& d_{A}=\frac{1}{2} d_{A B C} J^{B} J^{C} .
\end{aligned}
$$


In terms of these quantities, one easily finds

$$
\begin{aligned}
& \mathcal{V}_{i}=d_{A} J_{i}^{A}, \\
& \mathcal{V}_{i \bar{\jmath}}=d_{A B} J_{i}^{A} J_{\bar{\jmath}}^{B}+d_{A} J_{i \bar{\jmath}}^{A}, \\
& \mathcal{V}_{i \bar{\jmath} p}=d_{A B C} J_{i}^{A} J_{\bar{\jmath}}^{B} J_{p}^{C}+d_{A B}\left(J_{i \bar{\jmath}}^{A} J_{p}^{B}+J_{\bar{p} \bar{\jmath}}^{A} J_{i}^{B}\right), \\
& \mathcal{V}_{i \bar{\jmath} p \bar{q}}=d_{A B C}\left(J_{i \bar{\jmath}}^{A} J_{p}^{B} J_{\bar{q}}^{C}+3 \text { p. }\right)+d_{A B}\left(J_{i \bar{\jmath}}^{A} J_{p \bar{q}}^{B}+J_{i \bar{q}}^{A} J_{p \bar{\jmath}}^{B}\right), \\
& \mathcal{V}_{i \bar{\jmath} p \bar{q} n}=d_{A B C}\left(J_{i \bar{\jmath}}^{A} J_{p \bar{q}}^{B} J_{n}^{C}+5 \text { p. }\right) .
\end{aligned}
$$

Using these expressions and going to the canonical frame at the reference point, one then obtains the following non-vanishing entries for the derivatives of $Y=\mathcal{V}$ :

$$
\begin{aligned}
& Y_{A}=d_{A}, \\
& Y_{A \bar{B}}=d_{A B}, \quad Y_{\alpha \bar{\beta}}=-\delta_{\alpha \beta}, \\
& Y_{A \bar{B} C}=d_{A B C}, \quad Y_{A \alpha \bar{\beta}}=-d_{A X} c_{\alpha \beta}^{X}, \\
& Y_{A \bar{B} C \bar{D}}=0, \quad Y_{A \bar{B} \alpha \bar{\beta}}=-d_{A B X} c_{\alpha \beta}^{X}, \quad Y_{\alpha \bar{\beta} \gamma \bar{\delta}}=d_{X Y}\left(c_{\alpha \beta}^{X} c_{\gamma \delta}^{Y}+c_{\alpha \delta}^{X} c_{\gamma \beta}^{Y}\right), \\
& Y_{A \bar{B} C \bar{D} E}=0, \quad Y_{A \bar{B} C \alpha \bar{\beta}}=0, \quad Y_{A \alpha \bar{\beta} \gamma \bar{\delta}}=d_{A X Y}\left(c_{\alpha \beta}^{X} c_{\gamma \delta}^{Y}+c_{\alpha \delta}^{X} c_{\gamma \beta}^{Y}\right) .
\end{aligned}
$$

The parameters in these expressions are now given by $d_{A}=3 / 2 d_{A 00}, d_{A B}=\sqrt{3} d_{A B 0}$ and $d_{A B C}$ taking the already studied restricted form. Moreover, it will also be useful to define the following combinations of the parameters $d_{A B C}$ and $c_{\alpha \beta}^{A}$ :

$$
\begin{aligned}
& a_{A B C D}= \frac{1}{2}\left(d_{A B X} d_{X C D}+d_{A D X} d_{X B C}+d_{A C X} d_{X B D}\right) \\
&-\frac{1}{2}\left(d_{A B} d_{C D}+d_{A D} d_{B C}+d_{A C} d_{B D}\right) \\
&+\frac{1}{2}\left(d_{A} d_{B C D}+d_{B} d_{A C D}+d_{C} d_{A B D}+d_{D} d_{A B C}\right), \\
& b_{\alpha \beta}^{A B}=\frac{1}{2}\left(\left\{c^{A}, c^{B}\right\}_{\alpha \beta}-d_{A B X} c_{\alpha \beta}^{X}+d_{A B} \delta_{\alpha \beta}-d_{A} c_{\alpha \beta}^{B}-d_{B} c_{\alpha \beta}^{A}\right), \\
& \tau_{\alpha \beta \gamma \delta}^{A}=\frac{1}{2}\left(\left[c^{A}, c^{X}\right]_{\alpha \beta} c_{\gamma \delta}^{X}+\left[c^{A}, c^{X}\right]_{\alpha \delta} c_{\gamma \beta}^{X}+\left[c^{A}, c^{X}\right]_{\gamma \delta} c_{\alpha \beta}^{X}+\left[c^{A}, c^{X}\right]_{\gamma \beta} c_{\alpha \delta}^{X}\right) .
\end{aligned}
$$

These quantities are completely symmetric in their indices of type $A, B, \cdots$ and vanish if one of these is equal to 0 , meaning that $a_{A B C X} d_{X}=0, b_{\alpha \beta}^{A X} d_{X}=0$ and $\tau_{\alpha \beta \gamma \delta}^{X} d_{X}=0$.

Starting from the above expressions, it is now straightforward to compute all the geometric quantities we are interested in. The metric is trivial and its non-vanishing entries are

$$
\begin{aligned}
& g_{A \bar{B}}=\delta_{A B}, \\
& g_{\alpha \bar{\beta}}=\delta_{\alpha \beta} .
\end{aligned}
$$

The Christoffel connection is instead non-trivial, and its non-vanishing entries are

$$
\begin{aligned}
& \Gamma_{A B \bar{C}}=-d_{A B C}+\left(d_{A B} d_{C}+2 \text { p. }\right)-2 d_{A} d_{B} d_{C}, \\
& \Gamma_{A \alpha \bar{\beta}}=-c_{\alpha \beta}^{A} .
\end{aligned}
$$


The Riemann curvature tensor is found to be given by

$$
\begin{aligned}
& R_{A \bar{B} C \bar{D}}=\delta_{A B} \delta_{C D}+\delta_{A D} \delta_{B C}-d_{A C X} d_{X B D}, \\
& R_{\alpha \bar{\beta} \gamma \bar{\delta}}=c_{\alpha \beta}^{X} c_{\gamma \delta}^{X}+c_{\alpha \delta}^{X} c_{\gamma \beta}^{X}, \\
& R_{\alpha \bar{\beta} A \bar{B}}=\delta_{A B} \delta_{\alpha \beta}-d_{A X} d_{B Y}\left(c^{X} c^{Y}\right)_{\alpha \beta}+d_{A B X} c_{\alpha \beta}^{X} .
\end{aligned}
$$

Finally, the covariant derivative of the Riemann tensor reads

$$
\begin{aligned}
\nabla_{A} R_{B \bar{C} D \bar{E}}= & -2 a_{A B D X} d_{X C E} \\
\nabla_{A} R_{B \bar{C} \alpha \bar{\beta}}= & a_{A B C X} c_{\alpha \beta}^{X}+2 d_{C} b_{\alpha \beta}^{A B}-d_{A B X} b_{\alpha \beta}^{X C}+d_{A C X} b_{\alpha \beta}^{X B}+d_{B C X} b_{\alpha \beta}^{X A} \\
& -\left\{b^{A B}, c^{C}\right\}_{\alpha \beta}+\left[b^{A C}, c^{B}\right]_{\alpha \beta}+\left[b^{B C}, c^{A}\right]_{\alpha \beta}, \\
\nabla_{A} R_{\alpha \bar{\beta} \gamma \bar{\delta}}= & \tau_{\alpha \beta \gamma \delta}^{A}+b_{\alpha \beta}^{A X} c_{\gamma \delta}^{X}+b_{\alpha \delta}^{A X} c_{\gamma \beta}^{X}+b_{\gamma \delta}^{A X} c_{\alpha \beta}^{X}+b_{\gamma \beta}^{A X} c_{\alpha \delta}^{X} .
\end{aligned}
$$

We thus see that the manifold is a symmetric space with covariantly constant curvature if and only if the quantities $a_{A B C D}, b_{\alpha \beta}^{A B}$ and $\tau_{\alpha \beta \gamma \delta}^{A}$ identically vanish.

\section{Orientifold models}

Let us now consider the other specific case of orientifold string models based on a CalabiYau manifold $X$ with D7-branes supporting a non-trivial vector bundle and wrapping on some four-cycles $C$ of $X$ (see $[38,39]$ for a review). In this case, the Kähler moduli $T^{A}$ are associated to harmonic $(1,1)$ forms $\omega^{A}$ on $X$ which are dual to the harmonic $(2,2)$ forms $\omega_{A}$, while the matter fields $\Phi^{\alpha}$ are associated to bundle-valued harmonic $(1,0)$ forms $u_{\alpha}$ on $C \subset X$. Notice that for later convenience we use here opposite conventions compared to the heterotic case for the position of the index labeling harmonic forms and their duals. Denoting by $i$ the embedding map defining $C$ in $X$ and by $i^{*}$ its pullback on forms, the relevant numbers defining the low-energy effective theory are then given by the following integrals:

$$
\begin{aligned}
& d^{A B C}=\int_{X} \omega^{A} \wedge \omega^{B} \wedge \omega^{C}, \\
& c_{\alpha \beta}^{A}=\int_{C} i^{*} \omega^{A} \wedge \operatorname{tr}\left[u_{\alpha} \wedge \bar{u}_{\beta}\right] .
\end{aligned}
$$

We will again take the point of view that a priori $d^{A B C}$ can be an arbitrary symmetric symbol and similarly that $c_{\alpha \beta}^{A}$ can be an arbitrary set of Hermitian matrices, and study the low-energy effective scalar geometry as a function of these parameters.

\subsection{Kähler potential}

The effective Kähler potential for the Kähler moduli $T^{A}$ and the matter fields $\Phi^{\alpha}$ can, as before, be worked out by dimensionally reducing the kinetic terms of the ten-dimensional supergravity theory describing the unoriented string below the Planck scale down to four dimensions, retaining only the harmonic components of the fields. The full moduli and

matter field dependence was worked out in [40,41, 42], generalizing the results that were 
previously known for the special case of orbifold limits (see for example [39]). The complete result depends on the parameters $d^{A B C}$ and $c_{\alpha \beta}^{A}$ and takes again the form $K=-\log Y$, where the function $Y$ depends only on certain combinations of fields. More precisely, we have in this case

$$
Y=\mathcal{V}^{2}
$$

where $\mathcal{V}$ denotes the volume of the Calabi-Yau manifold and is given by the following expression in terms of the real geometric moduli fields $v_{A}$ :

$$
\mathcal{V}=\frac{1}{6} d^{A B C} v_{A} v_{B} v_{C}
$$

The real fields $v_{A}$ are then linked to the following real combination of complex fields:

$$
J^{A}=T^{A}+\bar{T}^{A}-c_{\alpha \beta}^{A} \Phi^{\alpha} \bar{\Phi}^{\beta} .
$$

The relation between the $v_{A}$ and the $J^{A}$ is in this case non-trivial and defined by the following equation:

$$
J^{A}=\frac{\partial \mathcal{V}}{\partial v_{A}}=\frac{1}{2} d^{A B C} v_{B} v_{C}
$$

It follows that in general $Y$ cannot be explicitly written in terms of the variables $J^{A}$ and is only implicitly defined:

$$
Y=Y(J)
$$

We see that $Y$ is as before a homogenous function of degree three in the variables $J^{A}$, and we therefore have again a no-scale manifold. However, the function $Y$ is in this case no-longer always a simple cubic polynomial in the variables $J^{A}$, and generically involves non-integer powers of them.

\subsection{Canonical parametrization}

We now want to study the above space at a given reference point, corresponding to the VEVs $\left\langle T^{A}\right\rangle$ and $\left\langle\Phi^{\alpha}\right\rangle$ that the scalar fields eventually acquire. For simplicity, we shall again restrict to the situation where the moduli have sizable VEVs whereas the matter fields have negligible VEVs, that is:

$$
\left\langle T^{A}\right\rangle \neq 0,\left\langle\Phi^{\alpha}\right\rangle=0 .
$$

For any given reference point of the type (4.8), it is again possible to define a particularly convenient canonical parametrization, in such a way as to simplify geometrical quantities at that point. To this aim, we proceed along the same lines as before and consider the field redefinitions

$$
\hat{T}^{A}=U_{B}^{A} T^{B}, \hat{\Phi}^{\alpha}=V_{\beta}^{\alpha} \Phi^{\beta},
$$

together with the parameter redefinitions

$$
\hat{d}^{A B C}=\alpha^{-1} U^{A}{ }_{D} U^{B}{ }_{E} U^{C}{ }_{F} d^{D E F}, \hat{c}_{\alpha \beta}^{A}=U_{B}^{A} V_{\alpha}^{-1 \gamma} \bar{V}_{\beta}^{-1 \delta} c_{\gamma \delta}^{B} .
$$


Under the above combined transformations, with $U_{B}^{A}$ a real matrix, $V_{\beta}^{\alpha}$ a complex matrix, and $\alpha$ a positive real number, the real geometrical moduli transform as $\hat{v}_{A}=\sqrt{\alpha} U^{-1 B}{ }_{A} v_{B}$, and the Kähler potential remains unchanged, modulo an irrelevant Kähler transformation:

$$
\hat{K}=K-\log \alpha .
$$

We may now choose $U_{B}^{A}$ and $V_{\beta}^{\alpha}$ such that the VEVs of the fields are aligned along just one direction, the VEV of the metric becomes diagonal, and the overall scale of these two quantities is set to some reference value. We may furthermore choose $\alpha$ to set the overall scale of the intersection numbers to a convenient value. More specifically, we shall require as before that in the new basis the reference point should be at

$$
\left\langle\hat{T}^{A}\right\rangle=\frac{\sqrt{3}}{2} \delta_{0}^{A}+i(\cdots),\left\langle\hat{\Phi}^{\alpha}\right\rangle=0
$$

the metric at that point should take the form

$$
\left\langle\hat{g}_{A \bar{B}}\right\rangle=\delta_{A B},\left\langle\hat{g}_{\alpha \bar{\beta}}\right\rangle=\delta_{\alpha \beta},\left\langle\hat{g}_{A \bar{\beta}}\right\rangle=0,
$$

and finally the Kähler frame should be such that at that point

$$
\langle\hat{K}\rangle=0 .
$$

It is again easy to get convinced that it is indeed always possible to impose this kind of conditions. Moreover, by proceeding as in the previous section, we deduce that the new values of $\hat{d}^{A B C}$ and $\hat{c}_{\alpha \beta}^{A}$ must satisfy the following properties:

$$
\begin{aligned}
& \hat{d}^{000}=\frac{2}{\sqrt{3}}, \quad \hat{d}^{00 a}=0, \quad \hat{d}^{0 a b}=-\frac{1}{\sqrt{3}} \delta_{a b}, \quad \hat{d}^{a b c}=\text { generic }, \\
& \hat{c}_{\alpha \beta}^{0}=\frac{1}{\sqrt{3}} \delta_{\alpha \beta}, \quad \hat{c}_{\alpha \beta}^{a}=\text { generic } .
\end{aligned}
$$

Notice finally that from the point of view of the Calabi-Yau manifold, the canonical frame just corresponds as before to a convenient choice of basis for harmonic forms, which is suitably oriented with respect to the Kähler form and normalized in such a way as to get unit volume, since $\left\langle\hat{v}_{A}\right\rangle=\sqrt{3} \delta_{A 0}$ and $\langle\hat{\mathcal{V}}\rangle=1$. Moreover, by comparing with the general results of section 2 we see that the canonical frame again essentially corresponds to choosing a parametrization such that the special direction $k^{i}$ is identified with one of the fields, since $\left\langle\hat{k}^{i}\right\rangle=\delta_{0}^{i}$.

\subsection{Geometry}

Let us now explore the geometry at a given reference point by using the new canonical coordinates. For notational simplicity, we drop from now on all the hats referring to the definition of this special frame, and also the brackets referring to the special point.

We start as before by computing the first five partial derivatives of $\mathcal{V}$, which are the basic ingredients that we need. In this case, there is an additional difficulty compared to the previous case, due to the fact that the relation between $J^{A}$ and $v_{A}$ cannot be explicitly inverted, in general. Fortunately, one can however get around this by just using 
the implicit definition of the $J^{A}$ in terms of the $v_{A}$. The Jacobian of this transformation is $\partial J^{A} / \partial v_{B}=d^{A B}$, where $d^{A B}=d^{A B C} v_{C}$, and its inverse is $\partial v_{A} / \partial J^{B}=\tilde{d}_{A B}$, where $\tilde{d}_{A B}$ is the inverse of the matrix $d^{A B}$. Let us also introduce the two new symbols $\tilde{d}_{A}=v_{A}$ and $\tilde{d}_{A B C}=\tilde{d}_{A E} \tilde{d}_{B F} \tilde{d}_{C G} d^{E F G}$. These satisfy simple algebraic properties: $\tilde{d}_{A B} J^{B}=1 / 2 \tilde{d}_{A}$ and $\tilde{d}_{A B C} J^{C}=1 / 2 \tilde{d}_{A B}$. Moreover, they depend on $J^{A}$ but their derivatives with respect to these variables have a very simple structure: $\partial \tilde{d}_{A} / \partial J^{B}=\tilde{d}_{A B}, \partial \tilde{d}_{A B} / \partial J^{C}=-\tilde{d}_{A B C}$ and $\partial \tilde{d}_{A B C} / \partial J^{D}=-\tilde{d}_{A B F} d^{F G} \tilde{d}_{G C D}-\tilde{d}_{A D F} d^{F G} \tilde{d}_{G B C}-\tilde{d}_{A C F} d^{F G} \tilde{d}_{G B D}$. It is then possible to express all derivatives of $\mathcal{V}$ in terms of the following quantities:

$$
\begin{aligned}
& \tilde{d}_{A}=v_{A}, d^{A}=\frac{1}{2} d^{A B C} v_{B} v_{C}, \\
& \tilde{d}_{A B}: \text { inverse of } d^{A B}=d^{A B C} v_{C}, \\
& \tilde{d}_{A B C}=\tilde{d}_{A E} \tilde{d}_{B F} \tilde{d}_{C G} d^{E F G} .
\end{aligned}
$$

After a straightforward computation, one finds that the first five derivatives of $\mathcal{V}$ can be written in the following form:

$$
\begin{aligned}
\mathcal{V}_{i}= & \frac{1}{2} \tilde{d}_{A} J_{i}^{A} \\
\mathcal{V}_{i \bar{\jmath}}= & \frac{1}{2} \tilde{d}_{A B} J_{i}^{A} J_{\bar{\jmath}}^{B}+\frac{1}{2} \tilde{d}_{A} J_{i \bar{\jmath}}^{A}, \\
\mathcal{V}_{i \bar{\jmath} p}= & -\frac{1}{2} \tilde{d}_{A B C} J_{i}^{A} J_{\bar{\jmath}}^{B} J_{p}^{C}+\frac{1}{2} \tilde{d}_{A B}\left(J_{i \bar{\jmath}}^{A} J_{p}^{B}+J_{p \bar{\jmath}}^{A} J_{i}^{B}\right) \\
\mathcal{V}_{i \bar{\jmath} p \bar{q}}= & \frac{1}{2} \tilde{d}_{A B X} d^{X Y} \tilde{d}_{Y C D}\left(J_{i}^{A} J_{\bar{\jmath}}^{B} J_{p}^{C} J_{\bar{q}}^{D}+2 \mathrm{p} .\right) \\
& -\frac{1}{2} \tilde{d}_{A B C}\left(J_{i}^{A} J_{\bar{\jmath}}^{B} J_{p \bar{q}}^{C}+3 \mathrm{p} .\right)+\frac{1}{2} \tilde{d}_{A B}\left(J_{i \bar{\jmath}}^{A} J_{p \bar{q}}^{B}+J_{i \bar{q}}^{A} J_{p \bar{\jmath}}^{B}\right), \\
\mathcal{V}_{i \bar{\jmath} p \bar{q} n}= & -\frac{1}{2} \tilde{d}_{A B X} d^{X Y} \tilde{d}_{Y E Z} d^{Z K} \tilde{d}_{K C D}\left(J_{i}^{A} J_{\bar{\jmath}}^{B} J_{p}^{C} J_{\bar{q}}^{D} J_{n}^{E}+14 \mathrm{p} .\right) \\
& +\frac{1}{2} \tilde{d}_{A B X} d^{X Y} \tilde{d}_{Y C D}\left(J_{i \bar{\jmath}}^{A} J_{p}^{B} J_{\bar{q}}^{C} J_{n}^{D}+17 \mathrm{p} .\right)-\frac{1}{2} \tilde{d}_{A B C}\left(J_{i \bar{\jmath}}^{A} J_{p \bar{q}}^{B} J_{n}^{C}+5 \mathrm{p} .\right) .(4 .)
\end{aligned}
$$

Using these expressions and going to the canonical frame at the reference point, one then obtains the following non-vanishing entries for the derivatives of $Y=\mathcal{V}^{2}$ :

$$
\begin{aligned}
& Y_{A}=d_{A}, \\
& Y_{A \bar{B}}=d_{A B}, \quad Y_{\alpha \bar{\beta}}=-\delta_{\alpha \beta}, \\
& Y_{A \bar{B} C}=d_{A B C}, \quad Y_{A \alpha \bar{\beta}}=-d_{A X} c_{\alpha \beta}^{X}, \\
& Y_{A \bar{B} C \bar{D}}=-2 a_{A B C D}, Y_{A \bar{B} \alpha \bar{\beta}}=-d_{A B X} c_{\alpha \beta}^{X}, Y_{\alpha \bar{\beta} \gamma \bar{\delta}}=d_{X Y}\left(c_{\alpha \beta}^{X} c_{\gamma \delta}^{Y}+c_{\alpha \delta}^{X} c_{\gamma \beta}^{Y}\right), \\
& Y_{A \bar{B} C \bar{D} E}=\left(a_{A B C X} d_{X D E}+9 \text { p. }\right)+2\left(a_{A B C D} d_{E}+4 \text { p. }\right), \\
& Y_{A \bar{B} C \alpha \bar{\beta}}=2 a_{A B C X} c_{\alpha \beta}^{X}, \quad Y_{A \alpha \bar{\beta} \gamma \bar{\delta}}=d_{A X Y}\left(c_{\alpha \beta}^{X} c_{\gamma \delta}^{Y}+c_{\alpha \delta}^{X} c_{\gamma \beta}^{Y}\right),
\end{aligned}
$$

The parameters in these expressions are now given by $d_{A}=3 / 2 d_{A 00}, d_{A B}=\sqrt{3} d_{A B 0}$ and $d_{A B C}$ taking the already studied restricted form. To simplify the notation, we have lowered all the indices in these quantities with the trivial metric at the reference point. Finally, we introduce as before for convenience the following combinations of the parameters $d_{A B C}$ 
and $c_{\alpha \beta}^{A}$ :

$$
\begin{aligned}
& a_{A B C D}= \frac{1}{2}\left(d_{A B X} d_{X C D}+d_{A D X} d_{X B C}+d_{A C X} d_{X B D}\right) \\
&-\frac{1}{2}\left(d_{A B} d_{C D}+d_{A D} d_{B C}+d_{A C} d_{B D}\right) \\
&+\frac{1}{2}\left(d_{A} d_{B C D}+d_{B} d_{A C D}+d_{C} d_{A B D}+d_{D} d_{A B C}\right), \\
& b_{\alpha \beta}^{A B}=\frac{1}{2}\left(\left\{c^{A}, c^{B}\right\}_{\alpha \beta}-d_{A B X} c_{\alpha \beta}^{X}+d_{A B} \delta_{\alpha \beta}-d_{A} c_{\alpha \beta}^{B}-d_{B} c_{\alpha \beta}^{A}\right), \\
& \tau_{\alpha \beta \gamma \delta}^{A}=\frac{1}{2}\left(\left[c^{A}, c^{X}\right]_{\alpha \beta} c_{\gamma \delta}^{X}+\left[c^{A}, c^{X}\right]_{\alpha \delta} c_{\gamma \beta}^{X}+\left[c^{A}, c^{X}\right]_{\gamma \delta} c_{\alpha \beta}^{X}+\left[c^{A}, c^{X}\right]_{\gamma \beta} c_{\alpha \beta}^{X}\right) .
\end{aligned}
$$

These quantities are completely symmetric in their indices of type $A, B, \cdots$ and vanish if one of these is equal to 0 , meaning that $a_{A B C X} d_{X}=0, b_{\alpha \beta}^{A X} d_{X}=0$ and $\tau_{\alpha \beta \gamma \delta}^{X} d_{X}=0$.

Starting from the above expressions, it is now straightforward to compute all the geometric quantities we are interested in. The metric is trivial and its non-vanishing entries are

$$
\begin{aligned}
& g_{A \bar{B}}=\delta_{A B}, \\
& g_{\alpha \bar{\beta}}=\delta_{\alpha \beta} .
\end{aligned}
$$

The Christoffel connection is instead non-trivial, and its non-vanishing entries are

$$
\begin{aligned}
& \Gamma_{A B \bar{C}}=-d_{A B C}+\left(d_{A B} d_{C}+2 \text { p. }\right)-2 d_{A} d_{B} d_{C}, \\
& \Gamma_{A \alpha \bar{\beta}}=-c_{\alpha \beta}^{A} .
\end{aligned}
$$

The Riemann curvature tensor is found to be given by

$$
\begin{aligned}
& R_{A \bar{B} C \bar{D}}=\delta_{A B} \delta_{C D}+\delta_{A D} \delta_{B C}-d_{A C X} d_{X B D}+2 a_{A B C D}, \\
& R_{\alpha \bar{\beta} \gamma \bar{\delta}}=c_{\alpha \beta}^{X} c_{\gamma \delta}^{X}+c_{\alpha \delta}^{X} c_{\gamma \beta}^{X}, \\
& R_{\alpha \bar{\beta} A \bar{B}}=\delta_{A B} \delta_{\alpha \beta}-d_{A X} d_{B Y}\left(c^{X} c^{Y}\right)_{\alpha \beta}+d_{A B X} c_{\alpha \beta}^{X} .
\end{aligned}
$$

Finally, the covariant derivative of the Riemann tensor reads:

$$
\begin{aligned}
\nabla_{A} R_{B \bar{C} D \bar{E}}= & -a_{A B D X} d_{X C E}+\left(a_{A C E X} d_{X B D}+a_{B C E X} d_{X A D}+a_{D C E X} d_{X A B}\right) \\
- & \left(a_{A B C X} d_{X D E}+a_{A D C X} d_{X B E}+a_{B D C X} d_{X A E}\right. \\
& \left.+a_{A B E X} d_{X D C}+a_{A D E X} d_{X B C}+a_{B D E X} d_{X A C}\right) \\
& -2\left(a_{A B D C} d_{E}+a_{A B D E} d_{C}\right), \\
\nabla_{A} R_{B \bar{C} \alpha \bar{\beta}}=- & a_{A B C X} c_{\alpha \beta}^{X}+2 d_{C} b_{\alpha \beta}^{A B}-d_{A B X} b_{\alpha \beta}^{X C}+d_{A C X} b_{\alpha \beta}^{X B}+d_{B C X} b_{\alpha \beta}^{X A} \\
- & \left\{b^{A B}, c^{C}\right\}_{\alpha \beta}+\left[b^{A C}, c^{B}\right]_{\alpha \beta}+\left[b^{B C}, c^{A}\right]_{\alpha \beta}, \\
\nabla_{A} R_{\alpha \bar{\beta} \gamma \bar{\delta}}= & \tau_{\alpha \beta \gamma \delta}^{A}+b_{\alpha \beta}^{A X} c_{\gamma \delta}^{X}+b_{\alpha \delta}^{A X} c_{\gamma \beta}^{X}+b_{\gamma \delta}^{A X} c_{\alpha \beta}^{X}+b_{\gamma \beta}^{A X} c_{\alpha \delta}^{X} .
\end{aligned}
$$

We thus see that the manifold is a symmetric space with covariantly constant curvature if and only if the quantities $a_{A B C D}, b_{\alpha \beta}^{A B}$ and $\tau_{\alpha \beta \gamma \delta}^{A}$ identically vanish, exactly as before. 


\section{General features of the geometry}

From the results of the previous two sections, we discover that the form of the tensors characterizing the geometry of the scalar manifolds of heterotic and orientifold models are very similar in the canonical frame. This similarity is best and most concisely exhibited by explicitly splitting the indices parallel and orthogonal to the special direction defined by the canonical frame: $A=0, a$. The parameters specifying the model and also the vacuum point are then summarized in the previously defined quantities $d_{a b c}$ and $c_{\alpha \beta}^{a}$, where we have again dropped the hats for simplicity. It is however useful and convenient to introduce some specific notation for various combinations of these parameters, which will turn out to play special roles in the following. Recall first that the parameters controlling the deviation from the coset situation have the same expressions (3.29), (3.30), (3.31) and (4.31), (4.32), (4.33) in both models, and their only common non-trivial components are those where all the indices are orthogonal:

$$
\begin{aligned}
& a_{a b c d}=\frac{1}{2}\left(d_{a b r} d_{r c d}+d_{a d r} d_{r b c}+d_{a c r} d_{r b d}\right)-\frac{1}{3}\left(\delta_{a b} \delta_{c d}+\delta_{a d} \delta_{b c}+\delta_{a c} \delta_{b d}\right), \\
& b_{\alpha \beta}^{a b}=\frac{1}{2}\left\{c^{a}, c^{b}\right\}_{\alpha \beta}-\frac{1}{3} \delta_{a b} \delta_{\alpha \beta}-\frac{1}{2} d_{a b r} c_{\alpha \beta}^{r}, \\
& \tau_{\alpha \beta \gamma \delta}^{a}=\frac{1}{2}\left(\left[c^{a}, c^{r}\right]_{\alpha \beta} c_{\gamma \delta}^{r}+\left[c^{a}, c^{r}\right]_{\alpha \delta} c_{\gamma \beta}^{r}+\left[c^{a}, c^{r}\right]_{\gamma \delta} c_{\alpha \beta}^{r}+\left[c^{a}, c^{r}\right]_{\gamma \beta} c_{\alpha \delta}^{r}\right) .
\end{aligned}
$$

Let us next introduce also some short-hand notation for the following additional combi-

nations of parameters, which will allow us to write the geometry in a nice and compact form:

$$
\begin{aligned}
& x_{a b c d}=\frac{1}{2}\left(d_{a b r} d_{r c d}+d_{a d r} d_{r b c}-d_{a c r} d_{r b d}\right)+\frac{2}{3}\left(\delta_{a b} \delta_{c d}+\delta_{a d} \delta_{b c}-\delta_{a c} \delta_{b d}\right), \\
& y_{\alpha \beta}^{a b}=\frac{1}{2}\left[c^{a}, c^{b}\right]_{\alpha \beta}-\frac{1}{3} \delta_{a b} \delta_{\alpha \beta}-\frac{1}{2} d_{a b r} c_{\alpha \beta}^{r} .
\end{aligned}
$$

Finally, in the applications that we will discuss in the last two sections, it will also be useful to define the following last couple of quantities:

$$
\begin{aligned}
& \alpha_{a b c d}=-\frac{1}{4}\left(d_{a b r} d_{r c d}+d_{a d r} d_{r b c}-2 d_{a c r} d_{r b d}\right)-\frac{1}{3}\left(\delta_{a b} \delta_{c d}+\delta_{a d} \delta_{b c}-2 \delta_{a c} \delta_{b d}\right), \\
& \beta_{\alpha \beta}^{a b}=\frac{1}{2}\left[c^{a}, c^{b}\right]_{\alpha \beta} .
\end{aligned}
$$

\subsection{Generic case}

Let us first consider generic models with generic values of the parameters $d_{a b c}$ and $c_{\alpha \beta}^{a}$. These correspond to generic choices of Calabi-Yau manifolds and holomorphic vector bundles over them. With the help of the above notation, we can make the results of the previous two sections more explicit and compare them more efficiently. The metric is in both cases simply

$$
\begin{aligned}
& g_{0 \overline{0}}=1, \quad g_{a \bar{b}}=\delta_{a b}, \\
& g_{\alpha \bar{\beta}}=\delta_{\alpha \beta} .
\end{aligned}
$$


The Christoffel connection is also identical in the two cases and given by

$$
\begin{aligned}
& \Gamma_{00 \overline{0}}=-\frac{2}{\sqrt{3}}, \quad \Gamma_{a b \overline{0}}=-\frac{2}{\sqrt{3}} \delta_{a b}, \quad \Gamma_{0 a \bar{b}}=-\frac{2}{\sqrt{3}} \delta_{a b}, \quad \Gamma_{a b \bar{c}}=-d_{a b c}, \\
& \Gamma_{0 \alpha \bar{\beta}}=-\frac{1}{\sqrt{3}} \delta_{\alpha \beta}, \quad \Gamma_{a \alpha \bar{\beta}}=-c_{\alpha \beta}^{a} .
\end{aligned}
$$

The Riemann tensor can instead be written in the following simple way, with the upper and lower signs applying respectively to heterotic and orientifold models:

$$
\begin{aligned}
& R_{0 \overline{0} \overline{0} \overline{0}}=\frac{2}{3}, \quad R_{0 \overline{0} a \bar{b}}=\frac{2}{3} \delta_{a b}, \quad R_{a \overline{0} b \overline{0}}=\frac{2}{3} \delta_{a b}, \quad R_{a \bar{b} c \overline{0}}=\frac{1}{\sqrt{3}} d_{a b c}, \\
& R_{a \bar{b} c \bar{d}}=x_{a b c d} \mp a_{a b c d}, \\
& R_{\alpha \bar{\beta} \gamma \bar{\delta}}=\frac{1}{3}\left(\delta_{\alpha \beta} \delta_{\gamma \delta}+\delta_{\alpha \delta} \delta_{\gamma \beta}\right)+c_{\alpha \beta}^{r} c_{\gamma \delta}^{r}+c_{\alpha \delta}^{r} c_{\gamma \beta}^{r}, \\
& R_{\alpha \bar{\beta} 0 \overline{0}}=\frac{1}{3} \delta_{\alpha \beta}, \quad R_{\alpha \bar{\beta} a \bar{b}}=-y_{\alpha \beta}^{a b}-b_{\alpha \beta}^{a b}, \quad R_{\alpha \bar{\beta} 0 \bar{b}}=\frac{1}{\sqrt{3}} c_{\alpha \beta}^{b} .
\end{aligned}
$$

Finally the covariant derivative of the Riemann tensor also differs only by a few signs for heterotic and orientifold models and reads:

$$
\begin{aligned}
& \nabla_{a} R_{b \bar{c} d \overline{0}}= \pm \frac{2}{\sqrt{3}} a_{a b c d} \\
& \nabla_{a} R_{b \bar{c} d \bar{e}}=-\frac{3 \pm 1}{2} a_{a b d r} d_{r c e}+\frac{1 \mp 1}{2}\left(a_{a c e r} d_{r b d}+2 \mathrm{p} .\right)-\frac{1 \mp 1}{2}\left(a_{a b c r} d_{r d e}+5 \mathrm{p} .\right) \\
& \nabla_{a} R_{b \overline{0} \alpha \bar{\beta}}=\frac{2}{\sqrt{3}} b_{\alpha \beta}^{a b} \\
& \nabla_{a} R_{b \bar{c} \alpha \bar{\beta}}=\left( \pm a_{a b c r} c^{r}-d_{a b r} b^{r c}+d_{a c r} b^{r b}+d_{b c r} b^{r a}-\left\{b^{a b}, c^{c}\right\}+\left[b^{a c}, c^{b}\right]+\left[b^{b c}, c^{a}\right]\right)_{\alpha \beta}, \\
& \nabla_{a} R_{\alpha \bar{\beta} \gamma \bar{\delta}}=\tau_{\alpha \beta \gamma \delta}^{a}+b_{\alpha \beta}^{a r} c_{\gamma \delta}^{r}+b_{\alpha \delta}^{a r} c_{\gamma \beta}^{r}+b_{\gamma \delta}^{a r} c_{\alpha \beta}^{r}+b_{\gamma \beta}^{a r} c_{\alpha \delta}^{r} .
\end{aligned}
$$

The space is thus generically not symmetric and becomes so if and only if the parameters $d_{a b c}$ and $c_{\alpha \beta}^{a}$ are such that $a_{a b c d}=0, b_{\alpha \beta}^{a b}=0$ and $\tau_{\alpha \beta \gamma \delta}^{a}=0$.

It is straightforward to show that for a given Calabi-Yau manifold, the scalar manifolds of the heterotic and the orientifold models coincide if and only if $a_{a b c d}=0$. In such a situation, we see from the formulae derived in previous section that the metric, the Christoffel connection, the Riemann tensor and the covariant derivative of the Riemann tensor do indeed coincide for the two models. In fact, one can easily check that in that case the whole Kähler potentials coincide for the two models. Indeed, it can be shown that the condition $a_{a b c d}=0$ which is equivalent to $a_{A B C D}=0$ also implies that $d_{X Y Z} d_{X(A B} d_{Y C D} d_{Z E F)}=4 / 3 d_{(A B C} d_{D E F)}$. This last relation then directly implies that $Y$ and thus $K$ coincide in the two models, as can be seen by comparing (3.7) and the square of (4.4) with the relation (4.6). This result generalizes a similar observation done in $[43,44]$ for models with only moduli fields to models involving also matter fields, with the significant difference that the coincidence of the scalar manifolds of the two kinds of models no-longer implies that they are symmetric spaces, since one may have $b_{\alpha \beta}^{a b} \neq 0$ and/or $\tau_{\alpha \beta \gamma \delta}^{a} \neq 0$. 


\subsection{Coset case}

Let us next discuss the special models with particular values of the parameters $d_{a b c}$ and $c_{\alpha \beta}^{a}$ such that not only $a_{a b c d}=0$ but also $b_{\alpha \beta}^{a b}=0$ and $\tau_{\alpha \beta \gamma \delta}^{a}=0$. These correspond to very special choices of Calabi-Yau manifold and holomorphic bundle over it, which can for instance naturally arise from orbifold constructions. In such a situation, the scalar manifolds of the heterotic and the orientifold models not only coincide but reduce to a coset manifold. It is therefore of some interest to understand when this can occur.

The possible solutions to the three equations $a_{a b c d}=0, b_{\alpha \beta}^{a b}=0$ and $\tau_{\alpha \beta \gamma \delta}^{a}=0$ can in principle be classified, and define a finite list of possibilities for such coset spaces, which must be a subclass of all the possible Kähler symmetric manifolds described in [25]. For models with only moduli fields, this classification has been explicitly carried out in $[37,45]$. The basic observation is that the condition $a_{a b c d}=0$ which can be rewritten as $a_{A B C D}=0$ is also essentially equivalent to the condition $d_{X Y E} d_{X(A B} d_{Y C D)}=4 / 3 \delta_{E(A} d_{B C D)}$. This is easier to study and its solutions were shown to correspond to all the possible special Kähler symmetric manifolds. For models with also matter fields, a similar classification is presumably possible. The basic missing ingredient would be a general study of the other condition $b_{\alpha \beta}^{a b}=0$, and also $\tau_{\alpha \beta \gamma \delta}^{a}=0$ although this last condition seems to be most of the times automatically satisfied (see the examples below) and we will therefore consider it on a different footing. The solutions plausibly provide most of the possible extensions of special Kähler symmetric manifolds to Kähler symmetric manifolds by the addition of matter fields besides moduli fields. We will however not attempt here to perform a complete classification.

It is rather straightforward and instructive to verify that the standard coset scalar manifolds arising in the simplest orbifold string models in the untwisted sector do indeed represent non-trivial solutions of the three equations $a_{a b c d}=0, b_{\alpha \beta}^{a b}=0$ and $\tau_{\alpha \beta \gamma \delta}^{a}=0$. To see this, let us assume that the matrices $c^{a}$ are all traceless and form a compact Lie algebra, so that $\left[c^{a}, c^{b}\right]=i f_{a b c} c^{c}$ with completely antisymmetric structure constants $f_{a b c}$. One then automatically gets $\tau_{\alpha \beta \gamma \delta}^{a}=0$. The condition $b_{\alpha \beta}^{a b}=0$ implies instead $\left\{c^{a}, c^{b}\right\}=d_{a b c} c^{c}+2 / 3 \delta_{a b} \mathbb{1}$, meaning that $d_{a b c}$ is the completely symmetric invariant symbol of this algebra. One then finds that $\operatorname{tr}\left(c^{a} c^{b}\right)=\kappa \delta_{a b}, f_{a b c}=-i \kappa^{-1} \operatorname{tr}\left(\left[c^{a}, c^{b}\right] c^{c}\right)$ and $d_{a b c}=\kappa^{-1} \operatorname{tr}\left(\left\{c^{a}, c^{b}\right\} c^{c}\right)$, where $\kappa=\operatorname{tr}(\mathbb{1}) / 3$. One finally has to impose the condition $a_{a b c d}=0$, and this dramatically reduces the possible algebras. The simplest possibility is the $S U(3)$ algebra generated by the $3 \times 3$ matrices $\lambda^{a}$, and one can then choose $c^{a}=\lambda^{a} \otimes \mathbb{1}_{k}$. Other similar solutions can then also be obtained by replacing $S U(3)$ with one of its maximal-rank subalgebras $S U(2) \times U(1)$ and $U(1) \times U(1)$. In this way, one obtains (see for example [19]) the following standard coset no-scale manifolds, with $m=8,4$ or 2 non-minimal moduli and $n=3 k, 2 k+k^{\prime}$ or $k+k^{\prime}+k^{\prime \prime}$ matter fields:

$$
\begin{aligned}
& \frac{S U(3,3+k)}{U(1) \times S U(3) \times S U(3+k)}, \\
& \frac{S U(2,2+k)}{U(1) \times S U(2) \times S U(2+k)} \times \frac{S U\left(1,1+k^{\prime}\right)}{U(1) \times S U\left(1+k^{\prime}\right)}, \\
& \frac{S U(1,1+k)}{U(1) \times S U(1+k)} \times \frac{S U\left(1,1+k^{\prime}\right)}{U(1) \times S U\left(1+k^{\prime}\right)} \times \frac{S U\left(1,1+k^{\prime \prime}\right)}{U(1) \times S U\left(1+k^{\prime \prime}\right)} .
\end{aligned}
$$


To conclude this section, let us discuss the meaning of the parameters $a_{a b c d}, b_{\alpha \beta}^{a b}$ and $\tau_{\alpha \beta \gamma \delta}^{a}$ in the simplest situations. In the trivial case where there is only one modulus and an arbitrary number of matter fields, the above quantities do not exist and one always gets a maximally symmetric coset space. The simplest non-trivial case is therefore when there are two moduli fields and one matter field, so that all the indices $a, c, \cdots$ and $\alpha, \beta, \cdots$ take a single value and can be dropped. In the canonical frame, and denoting for short $d=d_{111}$ and $c=c_{11}^{1}$, the definitions (5.1), (5.2) and (5.3) then give

$$
a=\frac{3}{2} d^{2}-1, \quad b=c^{2}-\frac{1}{2} d c-\frac{1}{3}, \quad \tau=0 .
$$

Notice also in passing that from (5.6) and (5.7) one gets $\alpha=0$ and $\beta=0$. One may then wonder whether it is possible to understand in a simple and intuitive way the origin and the meaning of the conditions $a=0$ and $b=0$, and perhaps work out their generalization to a generic frame. It turns out that this is indeed possible in this simple situation. The basic reason is that there is a unique candidate coset space for this type of models, which is $S U(1,2) /(U(1) \times S U(2)) \times S U(1,1) / U(1)$ and can be described by a Kähler potential of the form $K=-n^{\prime} \log \left(T^{\prime}+\bar{T}^{\prime}-\Phi^{\prime} \bar{\Phi}^{\prime}\right)-n^{\prime \prime} \log \left(T^{\prime \prime}+\bar{T}^{\prime \prime}\right)$ with $n^{\prime}+n^{\prime \prime}=3$. The conditions for getting a coset space in this class of models must then correspond to the conditions under which the Kähler potential $K=-\log \left[1 / 6 d_{A B C} J^{A} J^{B} J^{C}\right]$, where $J^{A}=T^{A}+\bar{T}^{A}-c_{\alpha \beta}^{A} \Phi^{\alpha} \bar{\Phi}^{\beta}$ with $A, B, \cdots=0,1$ and $\alpha, \beta, \cdots=1$, takes this simpler form, modulo a field redefinition from $T^{1}, T^{2}, \Phi^{1}$ to $T^{\prime}, T^{\prime \prime}, \Phi^{\prime}$ and a Kähler transformation. It is now straightforward to determine under which circumstances this is possible. A first condition is that the cubic polynomial defined by the intersection numbers $d_{A B C}$ factorize into two factors. This is possible if and only if the discriminant $\Delta$ of this polynomial vanishes, so that there is one real simple root $R_{1}$ and one real double root $R_{2}$, where:

$$
\begin{aligned}
& \Delta=-27\left(d_{000}^{2} d_{111}^{2}-3 d_{001}^{2} d_{011}^{2}+4 d_{000} d_{011}^{3}+4 d_{001}^{3} d_{111}-6 d_{000} d_{001} d_{011} d_{111}\right) \\
& R_{1}=-\frac{d_{001}}{d_{000}}-\frac{2}{d_{000}} \sqrt[3]{d_{001}^{3}-\frac{3}{2} d_{000} d_{001} d_{011}+\frac{1}{2} d_{000}^{2} d_{111}} \\
& R_{2}=-\frac{d_{001}}{d_{000}}+\frac{1}{d_{000}} \sqrt[3]{d_{001}^{3}-\frac{3}{2} d_{000} d_{001} d_{011}+\frac{1}{2} d_{000}^{2} d_{111}}
\end{aligned}
$$

In that case the Kähler potential factorizes into the sum of one trivial and two non-trivial pieces: $K=-\log \left[1 / 6 d_{000}\right]-\log \left[J^{0}-R_{1} J^{1}\right]-2 \log \left[J^{0}-R_{2} J^{1}\right]$. A second condition is then that the matter fields appear either in the second or the third term but not simultaneously in both. It is straightforward to check that this requires that either $c_{11}^{0}-R_{1} c_{11}^{1}$ or $c_{11}^{0}-R_{2} c_{11}^{1}$ vanishes, or equivalently that their product vanishes. To sum up, the two conditions for the space to degenerate into a coset are in this case:

$$
\begin{aligned}
& \Delta=0, \\
& \left(c_{11}^{0}-R_{1} c_{11}^{1}\right)\left(c_{11}^{0}-R_{2} c_{11}^{1}\right)=0 .
\end{aligned}
$$

It is now straightforward to verify that in the canonical frame one has $\Delta=-24 a$, and that whenever $a=0$ one finds $\left(c_{11}^{0}-R_{1} c_{11}^{1}\right)\left(c_{11}^{0}-R_{2} c_{11}^{1}\right)=-b$. This shows that the combination of the two conditions (5.28) and (5.29) is equivalent to the combination of the conditions $a=0$ and $b=0$ in the canonical frame, and evidently represents their generalization to 
arbitrary frames. From the above reasoning, it is however also clear that in the more general case where more than two moduli fields and/or more than one matter field are present, the situation is much more complicated to study from this frame-independent perspective. On the other hand, the conditions in the canonical frame simply generalize to the conditions $a_{a b c d}=0, b_{\alpha \beta}^{a b}=0$ and $\tau_{\alpha \beta \gamma \delta}^{a}=0$.

\section{SGoldstino mass and vacuum metastability}

As a first application of the results derived in the previous sections, let us consider the condition for the existence of a metastable supersymmetry breaking vacuum. This is controlled by the sign of the average sGoldstino square mass and depends on the holomorphic sectional curvature of the scalar manifold along the Goldstino direction $f^{I}$. More precisely, assuming for simplicity a negligibly small cosmological constant, the average sGoldstino mass is given by

$$
m_{f \bar{f}}^{2}=3\left(R(f)+\frac{2}{3}\right) m_{3 / 2}^{2},
$$

where the holomorphic sectional curvature $R(f)$ is defined as

$$
R(f)=-R_{I \bar{J} K \bar{L}} f^{I} \bar{f}^{\bar{J}} f^{K} \bar{f} \bar{L},
$$

and the vector $f^{I}$ is subject to the following constraint:

$$
|f|^{2}=g_{I \bar{J}} f^{I} \bar{f}^{\bar{J}}=1 .
$$

A necessary condition for metastability is that $m_{f \bar{f}}^{2}>0$, which implies $R(f)>-2 / 3$. This condition becomes also sufficient whenever the superpotential can be arbitrarily tuned, and the upper bound represented by $m_{f \bar{f}}^{2}$ on the square mass of the lightest particle can then be saturated. In the presence of a positive cosmological constant $V$ parametrized by

$\gamma=V /\left(3 m_{3 / 2}^{2}\right)$ this bound becomes stronger and reads $R(f)>-2 / 3(1+\gamma)^{-1}[46]$. The effect of vector multiplets has instead been studied in [47], and it has also been pointed out in [48] that in the presence of broken gauge symmetries the lightest scalar is in fact a combination of the sGoldstino and the complex partners of the Goldstones.

In the class of models that we considered, the hidden sector triggering supersymmetry breaking can involve both the dilaton $S$ and a subset of the Kähler moduli and matter fields $Z^{i}=T^{A}, \Phi^{\alpha}$. We can thus have $f^{S} \neq 0$ and $f^{i} \neq 0$. Since the dilaton sector and the Kähler moduli plus matter field sector are factorized, it is convenient to introduce an angle $\theta$ to explicitly parametrize the splitting of the Goldstino direction along the two corresponding submanifolds and rewrite $f^{S}=\sin \theta g^{S}$ and $f^{i}=\cos \theta h^{i}$, where now $|g|=1$ and $|h|=1$. We will imagine here that such a direction can a priori be arbitrary, as in [11, 12] (see also [13]), and shall not discuss the possibilities offered by specific effects like classical fluxes or non-perturbative quantum corrections (see for example $[49,50,51,52]$ ) for recent studies on this). Recalling that the sectional curvature of the fixed coset manifold $S U(1,1) / U(1)$ describing the dilaton is constant and equal to $R(g)=-2$, and parametrizing the sectional curvature of the generic no-scale manifold $\mathcal{M}_{Y, N}$ describing the Kähler moduli and matter fields as $R(h)=-2 / 3+\Sigma(h)$, one can then write $R(f)$ in the following form:

$$
R(f)=-2 \sin ^{4} \theta+\left(-\frac{2}{3}+\Sigma(h)\right) \cos ^{4} \theta .
$$


The average sGoldstino mass is correspondingly written as

$$
m_{f \bar{f}}^{2}=\left[-4 \sin ^{4} \theta+4 \sin ^{2} \theta \cos ^{2} \theta+3 \Sigma(h) \cos ^{4} \theta\right] m_{3 / 2}^{2} .
$$

The quantity $\Sigma(h)$ can be non-zero only if the no-scale manifold $\mathcal{M}_{Y, N}$ differs from the minimal possibility $S U(1,1+n) /(U(1) \times S U(n))$. It measures the amount by which the sectional curvature deviates from the critical value $-2 / 3$, and controls therefore the possibility of making $m_{f \bar{f}}^{2} \neq 0$ even when $\theta=0$. A quite explicit but still general expression for it can be derived by using the general properties of the geometry of no-scale manifolds derived in section 2, with $Y$ homogeneous of degree three in $J^{A}$ and $N^{A}$ function of $\Phi^{\alpha} \bar{\Phi}^{\beta}$, under the simplifying assumption that the matter fields take vanishing expectation values. Using the short-hand notation in which at the considered point the moduli index $A$ is split into the values 0 corresponding to the direction parallel to $k^{A}$ and the values $a$ corresponding to the directions orthogonal to $k^{A}$, and reading off the values of the metric, the Christoffel symbol and the Riemann tensor with at least one parallel index from eqs. (2.27)-(2.35), one then finds:

$$
\Sigma(h)=A_{a \bar{b} c \bar{d}} h^{a} \bar{h}^{\bar{b}} h^{c} \bar{h}^{\bar{d}}+4 B_{a \bar{b} \alpha \bar{\beta}} h^{a} \bar{h}^{\bar{b}} h^{\alpha} \bar{h}^{\bar{\beta}}+E_{\alpha \bar{\beta} \gamma \bar{\delta}} h^{\alpha} \bar{h}^{\bar{\beta}} h^{\gamma} \bar{h}^{\bar{\delta}}-2 S^{r}(h) S_{r}(h) .
$$

where

$$
\begin{aligned}
& A_{a \bar{b} c \bar{d}}=\frac{1}{3}\left(g_{a \bar{b}} g_{c \bar{d}}+g_{a \bar{d}} g_{c \bar{b}}\right)-R_{a \bar{b} c \bar{d}}+\frac{1}{4}\left(\Gamma_{r a \bar{b}} g^{r \bar{s}} \Gamma_{\bar{s} \bar{d} c}+\Gamma_{r a \bar{d}} g^{r \bar{s}} \Gamma_{\bar{s} \bar{b} c}\right) \\
& B_{a \bar{b} \alpha \bar{\beta}}=\frac{1}{3} g_{a \bar{b}} g_{\alpha \bar{\beta}}-R_{a \bar{b} \alpha \bar{\beta}}+\frac{1}{2} \Gamma_{r a \bar{b}} g^{r \bar{s}} \Gamma_{\bar{s} \bar{\beta} \alpha}, \\
& E_{\alpha \bar{\beta} \gamma \bar{\delta}}=\frac{1}{3}\left(g_{\alpha \bar{\beta}} g_{\gamma \bar{\delta}}+g_{\alpha \bar{\delta}} g_{\gamma \bar{\beta} \bar{\beta}}\right)-R_{\alpha \bar{\beta} \gamma \bar{\delta}}+\Gamma_{r \alpha \bar{\beta}} g^{r \bar{s}} \Gamma_{\bar{s} \bar{\delta} \gamma}+\Gamma_{r \alpha \bar{\delta}} g^{r \bar{s}} \Gamma_{\bar{s} \bar{\beta} \gamma}
\end{aligned}
$$

and

$$
S_{r}(h)=\frac{1}{\sqrt{3}}\left(h_{\bar{r}} \bar{h}^{\overline{0}}+\bar{h}_{r} h^{0}\right)-\frac{1}{2}\left(\Gamma_{r a \bar{b}} h^{a} \bar{h}^{\bar{b}}+2 \Gamma_{r \alpha \bar{\beta}} h^{\alpha} \bar{h}^{\bar{\beta}}\right) .
$$

The explicit form of the normalization condition for $h$ is:

$$
\left|h^{0}\right|^{2}+g_{a \bar{b}} h^{a} \bar{h}^{\bar{b}}+g_{\alpha \bar{\beta}} h^{\alpha} \bar{h}^{\bar{\beta}}=1 .
$$

Using the same strategy as in [20], we now observe that a simple bound on $\Sigma(h)$ can be obtained by dropping the sum of squares in the last term, which give negative-definite contributions, and keeping the first three terms, which have a priori indefinite signs. A necessary condition for the existence of any direction $h$ along which $\Sigma(h)$ is larger than 0 is then that the sum of these first three terms be larger than 0 for some $h$. In fact, the maximal value $\Sigma_{\text {up }}$ of the sum of these three terms represents an upper bound on how big the full $\Sigma(h)$ can be, and thus on its maximum $\Sigma_{\max }$. We thus deduce that

$$
\Sigma(h) \leq \Sigma_{\max } \leq \Sigma_{\text {up }}
$$

where:

$$
\Sigma_{\mathrm{up}}=\max _{h}\left\{A_{a \bar{b} c \bar{d}} h^{a} \bar{h}^{\bar{b}} h^{c} \bar{h}^{\bar{d}}+4 B_{a \bar{b} \alpha \bar{\beta}} h^{a} \bar{h}^{\bar{b}} h^{\alpha} \bar{h}^{\bar{\beta}}+E_{\alpha \bar{\beta} \gamma \bar{\delta}} h^{\alpha} \bar{h}^{\bar{\beta}} h^{\gamma} \bar{h}^{\bar{\delta}}\right\}
$$


We now want to evaluate more explicitly the quantities $\Sigma(h)$ and $\Sigma_{\text {up }}$ in the specific cases of Calabi-Yau string models of the heterotic and orientifold types, where the Riemann tensor and the Christoffel connection have a more constrained form parametrized in terms of some numbers $d_{A B C}$ and $c_{\alpha \beta}^{A}$. To do so, it is very convenient to go to the canonical frame defined in sections 3 and 4 . In this way, one can use the simple characterization of the geometry derived in section 5 , and after a straightforward computation one finds that the quantities (6.6), (6.7) and (6.8) reduce to the following combinations of the quantities (5.1), (5.2), (5.6) and (5.7):

$$
A_{a \bar{b} c \bar{d}}= \pm a_{a b c d}+\alpha_{a b c d}, \quad B_{a \bar{b} \alpha \bar{\beta}}=b_{\alpha \beta}^{a b}+\beta_{\alpha \beta}^{a b}, \quad E_{\alpha \bar{\beta} \gamma \bar{\delta}}=0 .
$$

One then finds

$$
\begin{aligned}
\Sigma(h)= & \left( \pm a_{a b c d}+\alpha_{a b c d}\right) h^{a} \bar{h}^{\bar{b}} h^{c} \bar{h}^{\bar{d}}+4\left(b_{\alpha \beta}^{a b}+\beta_{\alpha \beta}^{a b}\right) h^{a} \bar{h}^{\bar{b}} h^{\alpha} \bar{h}^{\bar{\beta}} \\
& -2 \sum_{a}\left[\frac{1}{\sqrt{3}}\left(\bar{h}^{\overline{0}} h^{a}+h^{0} \bar{h}^{\bar{a}}\right)+\frac{1}{2}\left(d_{a b c} h^{b} \bar{h}^{\bar{c}}+2 c_{\alpha \beta}^{a} h^{\alpha} \bar{h}^{\bar{\beta}}\right)\right]^{2},
\end{aligned}
$$

and thus

$$
\Sigma_{\text {up }}=\max _{h}\left\{\left( \pm a_{a b c d}+\alpha_{a b c d}\right) h^{a} \bar{h}^{\bar{b}} h^{c} \bar{h}^{\bar{d}}+4\left(b_{\alpha \beta}^{a b}+\beta_{\alpha \beta}^{a b}\right) h^{a} \bar{h}^{\bar{b}} h^{\alpha} \bar{h}^{\bar{\beta}}\right\} .
$$

We see that the structure of $\Sigma(h)$ is very similar in heterotic and orientifold models, the only difference being the sign with which the $a$ parameter related to moduli enters, as already noticed in [20], while the $b$ parameter related to matter fields enters with the same sign. The average sGoldstino mass correspondingly also takes very similar forms. We further notice that $\Sigma(h)$ has a very simple dependence on $h^{0}$, while the functional defining $\Sigma_{\text {up }}$ does not depend at all on $h^{0}$. This results in two distinct behaviors for directions $h^{i}$ that are parallel and orthogonal to $k^{i}$. In the parallel direction defined by taking $h^{0}=1$ and $h^{a}, h^{\alpha}=0$, one finds a trivially vanishing $\Sigma(h)$. In the orthogonal directions defined by taking $h^{0}=0$ and $h^{a}, h^{\alpha} \neq 0$, one instead finds a generically nontrivial and potentially positive $\Sigma(h)$. Notice also that in hybrid directions where $h^{a}=0$ and $h^{0}, h^{\alpha} \neq 0$, one finds again a vanishing $\Sigma(h)$ if the further constraints $c_{\alpha \beta}^{a} h^{\alpha} \bar{h}^{\bar{\beta}}=0$ hold true. In such a situation, the sGoldstino mass would then be given by the following bounded expression:

$$
m_{f \bar{f}}^{2}=\left[-4 \sin ^{4} \theta+4 \sin ^{2} \theta \cos ^{2} \theta\right] m_{3 / 2}^{2} \text { if } h^{a}=c_{\alpha \beta}^{a} h^{\alpha} \bar{h}^{\bar{\beta}}=0 .
$$

The above remarks also show that $\Sigma_{\mathrm{up}} \geq 0$, because the functional involved in the expression (6.15) always takes a vanishing value along the parallel and the hybrid directions and possibly a positive value along some orthogonal directions.

\subsection{Necessary conditions for metastability}

A first non-trivial question about $\Sigma(h)$ is to determine whether it can be positive, since this would allow for the existence of metastable supersymmetry breaking vacua even when the dilaton does not contribute to supersymmetry breaking. In the hope of finding some simple necessary conditions for this, one may then try to compute the sign of the associated 
$\Sigma_{\text {up }}$ and determine for which models it can be positive. To proceed, we parametrize the complex Goldstino variables in terms of a modulus and a phase, as $h^{i}=\tilde{h}^{i} e^{i \delta^{i}}$. We then notice that when all the phases vanish, the terms involving the quantities $\alpha_{a b c d}$ and $\beta_{\alpha \beta}^{a b}$ drop out, and the functional problem substantially simplifies. Let us then restrict to the situation where we deliberately fix $\delta^{i}=0$ and optimize only with respect to the real variables $\tilde{h}^{i}$, subject to the constraint $\sum_{i}\left(\tilde{h}^{i}\right)^{2}=1$. This defines the new quantity

$$
\tilde{\Sigma}_{\text {up }}=\max _{\tilde{h}}\left\{ \pm a_{a b c d} \tilde{h}^{a} \tilde{h}^{b} \tilde{h}^{c} \tilde{h}^{d}+4 b_{\alpha \beta}^{a b} \tilde{h}^{a} \tilde{h}^{b} \tilde{h}^{\alpha} \tilde{h}^{\beta}\right\} .
$$

Clearly the extremum $\tilde{\Sigma}_{\text {up }}$ of the simplified functional for real $\tilde{h}^{i}$ will in general be smaller than the extremum $\Sigma_{\text {up }}$ of the full original functional for complex $h^{i}$. But it is legitimate to hope that the gross behavior of $\tilde{\Sigma}_{\text {up }}$ as a function of the parameters of the models could still give a reasonable indication of the gross behavior of $\Sigma_{\mathrm{up}}$. We have numerically checked in some simple classes of models that this is indeed the case. We will therefore perform an analytical study of the properties of the simplified $\tilde{\Sigma}_{\text {up }}$ and then give a qualitative discussion of the properties of the true $\Sigma_{\text {up }}$.

To discuss the maximization defining $\tilde{\Sigma}_{\text {up }}$, it is convenient to introduce two angles $\chi$ and $\xi$ to explicitly parametrize the distribution of the real Goldstino direction $\tilde{h}^{i}$ along the three different types of fields arising in the no-scale sector and rewrite $\tilde{h}^{0}=\cos \chi \tilde{x}^{0}$, $\tilde{h}^{a}=\sin \chi \cos \xi \tilde{y}^{a}$ and $\tilde{h}^{\alpha}=\sin \chi \sin \xi \tilde{z}^{\alpha}$, where now $|\tilde{x}|=1,|\tilde{y}|=1$ and $|\tilde{z}|=1$. In this way, one can rewrite

$$
\tilde{\Sigma}_{\text {up }}=\max _{\chi, \xi, \tilde{y}, \tilde{z}}\left\{\sin ^{4} \chi\left( \pm a(\tilde{y}) \cos ^{4} \xi+4 b(\tilde{y}, \tilde{z}) \cos ^{2} \xi \sin ^{2} \xi\right)\right\},
$$

where

$$
a(\tilde{y})=a_{a b c d} \tilde{y}^{a} \tilde{y}^{b} \tilde{y}^{c} \tilde{y}^{d}, \quad b(\tilde{y}, \tilde{z})=b_{\alpha \beta}^{a b} \tilde{y}^{a} \tilde{y}^{b} \tilde{z}^{\alpha} \tilde{z}^{\beta} .
$$

It becomes now obvious that $\tilde{\Sigma}_{\text {up }}$ can be positive only if $\pm a(\tilde{y})$ or $b(\tilde{y}, \tilde{z})$ can be positive. We therefore conclude that:

$$
\tilde{\Sigma}_{\text {up }}>0 \text { requires }( \pm a)_{\text {up }} \equiv \max _{\tilde{y}}\{ \pm a(\tilde{y})\}>0 \text { or } b_{\text {up }} \equiv \max _{\tilde{y}, \tilde{z}}\{b(\tilde{y}, \tilde{z})\}>0 .
$$

For fixed $\tilde{y}^{a}$ and $\tilde{z}^{\alpha}$ and thus $a$ and $b$, the extrema of the functionals $F(\chi)=\sin ^{4} \chi$ and $G(\xi)= \pm a \cos ^{4} \xi+4 b \cos ^{2} \xi \sin ^{2} \xi$ are straightforward to find. For $F(\chi)$, the are always two extrema: the first is at $\chi=0$ and gives $F=0$, the second is at $\chi=\pi / 2$ and gives $F=1$. For $G(\xi)$ there are in general three extrema: the first is at $\xi=0$ and gives $G= \pm a$, the second is at $\xi=\pi / 2$ and gives $G=0$, the third is at $\chi=\arccos \sqrt{2 b /(4 b \mp a)}$ and leads to $G=4 b^{2} /(4 b \mp a)$. Notice however that while the first and the second of these always exist, the third exists when $b \notin] \min \{0, \pm a / 2\}, \max \{0, \pm a / 2\}[$ implying that $4 b^{2} /(4 b \mp a) \leq \max \{0,2 b\}$ but not when $\left.b \in\right] \min \{0, \pm a / 2\}, \max \{0, \pm a / 2\}[$ implying anyhow that $4 b^{2} /(4 b \mp a) \leq \max \{0, \pm a\}$. These results confirm that the maximum of the product functional $F(\chi) G(\xi)$ can be positive only if $a>0$ or $b>0$, while it is zero if $a \leq 0$ and $b \leq 0$. Moreover, they allow to compute the precise value of $\tilde{\Sigma}_{\text {up }}$ and to derive a simple bound on it. With an obvious notation one finds:

$$
\begin{aligned}
\tilde{\Sigma}_{\mathrm{up}} & =\max \left\{0,( \pm a)_{\mathrm{up}},\left(\frac{4 b^{2}}{4 b \mp a}\right)_{\mathrm{up}}\right\} \\
& \leq \max \left\{0,( \pm a)_{\mathrm{up}}, 2 b_{\mathrm{up}}\right\} .
\end{aligned}
$$


This concludes our analytic study of the necessary conditions for metastability in the approximation in which the sGoldstino direction is assumed to be real and without relying on the dilaton. The result (6.21) shows that for coset spaces where $a_{a b c d}=0$ and $b_{\alpha \beta}^{a b}=0$ one always finds $\tilde{\Sigma}_{\text {up }}=0$ since $( \pm a)_{\text {up }}=0$ and $b_{\text {up }}=0$, while for non-coset spaces where $a_{a b c d} \neq 0$ or $b_{\alpha \beta}^{a b} \neq 0$ one can obtain $\tilde{\Sigma}_{\text {up }}>0$ only if $( \pm a)_{\text {up }}>0$ or $b_{\text {up }}>0$. This generalizes the result that was derived in [20] (see also $[46,53]$ ) for models with two moduli and no matter fields to models with an arbitrary number of moduli and matter fields.

The maximization defining the true $\Sigma_{\text {up }}$ is more complicated, but as already anticipated its behavior is qualitatively similar to that of $\tilde{\Sigma}_{\text {up }}$, as a matter of fact. The main difference is that the necessary condition to get $\Sigma_{\text {up }}>0$ is not exactly given by a sharp conditions related just to $a_{a b c d}$ and $b_{\alpha \beta}^{a b}$, but is slightly affected also by $\alpha_{a b c d}$ and $\beta_{\alpha \beta}^{a b}$. More precisely, one finds a slight blurring of the sharp conditions that were required for $\tilde{\Sigma}_{\text {up }}>0$, and the requirements $( \pm a)_{\text {up }} \gtrsim 0$ or $b_{\text {up }} \gtrsim 0$ are now only qualitatively true far away from the transition points where $( \pm a)_{\text {up }} \sim 0$ or $b_{\text {up }} \sim 0$. This blurring is however quite limited and not always efficient. For instance, in the particular case of coset spaces, where $a_{a b c d}=0$ and $b_{\alpha \beta}^{a b}=0$ but in general $\alpha_{a b c d} \neq 0$ and $\beta_{\alpha \beta}^{a b} \neq 0$, one manifestly has $\tilde{\Sigma}_{\text {up }}=0$, but as a matter of fact one can verify case by case that one also finds $\Sigma_{\text {up }}=0$ and in fact $\Sigma_{\max }=0$, as was first derived in [17] for a large class of examples. In that case, we therefore see that the absence of the terms involving $a_{a b c d}$ and $b_{\alpha \beta}^{a b}$ is the crucial feature, while the presence of the terms involving $\alpha_{a b c d}$ and $\beta_{\alpha \beta}^{a b}$ is essentially irrelevant, in the sense that the maximal value of $\Sigma_{\text {up }}$ is realized along directions such that these terms vanish. More in general, we found evidence through numerical investigations that also for generic non-coset spaces the crucial features are again controlled by $a_{a b c d}$ and $b_{\alpha \beta}^{a b}$, while the presence of the terms involving $\alpha_{a b c d}$ and $\beta_{\alpha \beta}^{a b}$ gives only small effects. To sum up, this leads us to argue that if neither $( \pm a)_{\text {up }}$ nor $b_{\text {up }}$ are positive then the average sGoldstino mass is essentially bounded:

$$
m_{f \bar{f}}^{2} \lesssim\left[-4 \sin ^{4} \theta+4 \sin ^{2} \theta \cos ^{2} \theta\right] m_{3 / 2}^{2} \text { when }( \pm a)_{\text {up }}, b_{\text {up }} \lesssim 0 .
$$

In particular, in such a situation one would get $m_{f \bar{f}}^{2} \lesssim-4 m_{3 / 2}^{2}$ in the dilaton dominated case, $m_{f \bar{f}}^{2} \lesssim 0$ in the moduli dominated case, and $m_{f \bar{f}}^{2} \lesssim 1 / 2 m_{3 / 2}^{2}$ in any case.

Let us finally discuss the meaning of the signs of $( \pm a)_{\text {up }}$ and $b_{\text {up }}$ in the generic frame in which the model is defined. Recall first that the quantities $d_{a b c}$ and $c_{\alpha \beta}^{a}$ defined in the canonical frame implicitly depend on the original parameters $d_{A B C}$ and $c_{\alpha \beta}^{A}$ as well as on the reference point $P$. The same is therefore true also for the quantities $\pm a_{a b c d}$ and $b_{\alpha \beta}^{a b}$ as well as their extrema $( \pm a)_{\text {up }}$ and $b_{\text {up }}$. A crucial question is then whether the signs of $( \pm a)_{\text {up }}$ and $b_{\text {up }}$ are fixed within a given model specified by a choice of parameters $d_{A B C}$ and $c_{\alpha \beta}^{A}$ or can instead be changed by changing the point $P$ within the given model. To try to answer this question, we notice that the generalizations of the quantities $\pm a_{a b c d}$ and $b_{\alpha \beta}^{a b}$ defined in the canonical frame to an arbitrary frame are essentially given by the quantities $A_{a \bar{b} c \bar{d}}$ and $B_{a \bar{b} \alpha \bar{\beta}}$ defined in (6.6) and (6.7), at least if one ignores the effects of the terms involving $\alpha_{a b c d}$ and $\beta_{\alpha \beta}^{a b}$. This shows that the behavior of $\pm a_{a b c d}$ is sensitive to moduli but not matter fields, while the behavior of $b_{\alpha \beta}^{a b}$ depends both on moduli and matter fields. One can then try to evaluate more explicitly these expressions to understand how they are allowed to depend on $P$. In the simplest non-trivial case of models with two 
moduli fields and one matter field, this can indeed be done rather explicitly thanks to the fact that all the indices can take a single value and can thus be dropped. One is then left with just two parameters $A$ and $B$ which are in one-to-one correspondence with the parameters $\pm a$ and $b$ controlling the deviations from the coset space situation (since in this case $\tau=0$ ), and this allows to argue quite sharply about what kind of dependence on $P$ is allowed. We first notice that the sign of $A$ cannot change when continuously changing $P$. Indeed, if $A=0$ for some $P$ then it is effectively as if one had a coset space, since when studying $A$ one can focus on moduli fields and completely ignore matter fields so that the value of $B$ does not matter, and one must then get $A=0$ at any other $P$ too. This statement can be explicitly checked. Indeed, a straightforward computation shows that $A=-\Delta / 24 e^{4 K} \operatorname{det}^{-3} g$, where $\Delta$ is given by (5.25) and the remaining factor depends on $P$ but is not allowed to change sign. We next notice that the sign of $B$ can instead change when continuously changing $P$, because even if $B=0$ at some point $P$ one does not necessarily have a coset space, since when studying $B$ one must consider both the moduli and the matter fields so that the value of $A$ matters. If however one starts from a situation where $A=0$, then even $B$ is no longer allowed to change sign by continuously changing $P$, because if $B=0$ for some $P$ one has a coset space and one must then have $B=0$ also at any other $P$. These statements can be verified numerically, but we were not able to find any simple universal expression for $B$ that could make them manifest. In more general situations with more than two moduli fields and/or more than one matter field, the situation is clearly more complicated, since there are more parameters. It is then a priori always possible that $A(y)$ and $B(y, z)$ change sign when changing continuously $P$, because this does not imply that all the components of $A_{a \bar{b} c \bar{d}}$ and $B_{a \bar{b} \alpha \bar{\beta}}$ go through zero simultaneously. In other words, in this more general case the coset space situations do no longer separate the parameter space into semi-disconnected parts.

\subsection{Upper bound on the mass of the lightest scalar}

A second non-trivial question about $\Sigma(h)$ is to compute the maximal value $\Sigma_{\max }$ that it is allowed to take, since this allows to set an upper bound on the mass of the lightest scalar relative to $m_{3 / 2}$ which can have relevant cosmological implications (see for instance [46, 55]). To facilitate the discussion, we again introduce two angles $\chi$ and $\xi$ and parametrize the complex Goldstino direction $h^{i}$ in the usual form $h^{0}=\cos \chi x^{0}, h^{a}=\sin \chi \cos \xi y^{a}$ and $h^{\alpha}=\sin \chi \sin \xi z^{\alpha}$, where $|x|=1,|y|=1$ and $|z|=1$.

A general preliminary information that can be easily extracted concerns the absolute maximum that can be achieved for $\Sigma(h)$ within each class of models by suitably dialing not only the Goldstino direction $h^{i}$ but also the parameters $d_{a b c}$ and $c_{\alpha \beta}^{a}$. To derive such an absolute bound, we note that from the definitions of $a_{a b c d}, \alpha_{a b c d}, b_{\alpha \beta}^{a b}$ and $\beta_{\alpha \beta}^{a b}$ it follows that in eq. (6.14) the first term involving $\pm a_{a b c d}+\alpha_{a b c d}$ can be arbitrarily large in the heterotic case but at most unity in the orientifold case, while the second term involving $b_{\alpha \beta}^{a b}+\beta_{\alpha \beta}^{a b}$ can be arbitrarily large in both cases. This means that when only moduli fields participate in supersymmetry breaking one gets $\Sigma(h)<+\infty$ for heterotic models but $\Sigma(h)<1$ for orientifold models. On the other hand, when also matter fields participate in supersymmetry breaking one gets $\Sigma(h)<+\infty$ both for heterotic and orientifold models, and the situation therefore significantly improves. These extreme values of $\Sigma(h)$ can be 
obtained when the parameters $d_{a b c}$ and $c_{\alpha \beta}^{a}$ are either very large or very small, implying that $a_{a b c d}$ and $b_{\alpha \beta}^{a b}$ are necessarily non-zero and the model is thus far away from any coset. By studying these limits one can then determine more explicitly the behavior of $\Sigma(h)$ and its maximum $\Sigma_{\max }$ in these asymptotic regions.

Let us first consider the case where some of the parameters $d_{a b c}$ and $c_{\alpha \beta}^{a}$ are large. In such a situation one may keep only those terms in (6.14) that involve two powers of the parameters $d_{a b c}$ and $c_{\alpha \beta}^{a}$. This leads to the following expression:

$$
\begin{aligned}
\Sigma(\chi, \xi, y, z) \simeq \sin ^{4} \chi & {\left[\sum_{r}\left(-(1 \mp 1)\left(d_{r}(y)\right)^{2}+\frac{1 \pm 1}{2}\left|\hat{d}_{r}(y)\right|^{2}\right) \cos ^{4} \xi\right.} \\
& +4\left(\sum_{\epsilon}\left|\hat{c}_{\epsilon}(y, z)\right|^{2}-\sum_{r} d_{r}(y) c^{r}(z)\right) \cos ^{2} \xi \sin ^{2} \xi \\
& \left.-2 \sum_{r}\left(c^{r}(z)\right)^{2} \sin ^{4} \xi\right],
\end{aligned}
$$

where

$$
\begin{array}{ll}
d_{r}(y)=d_{r a b} y^{a} \bar{y}^{\bar{b}}, & \hat{d}_{r}(y)=d_{r a b} y^{a} y^{b}, \\
c^{r}(z)=c_{\alpha \beta}^{r} z^{\alpha} \bar{z}^{\bar{\beta}}, & \hat{c}_{\epsilon}(y, z)=c_{\alpha \epsilon}^{a} y^{a} z^{\alpha} .
\end{array}
$$

In this regime, the maximization of $\Sigma$ with respect to the angles $\chi$ and $\xi$ can be performed explicitly. When $|d| \gg|c|$, only the terms quadratic in $d_{a b c}$ matter, and we see that these are positive for heterotic models and negative for orientifold models. The maximum $\Sigma_{\max }$ is then obtained for $\chi=\pi / 2$ and $\xi=0$ in heterotic models and for $\chi=0$ in orientifold models:

$$
\Sigma_{\max } \simeq\left\{\begin{array}{l}
\max _{y}\left\{\sum_{r}\left|\hat{d}_{r}(y)\right|^{2}\right\} \quad \text { (heterotic) }, \\
0 \text { (orientifold) } .
\end{array}\right.
$$

When on the contrary $|c| \gg|d|$, only the terms that are quadratic in $c_{\alpha \beta}^{a}$ matter. The maximum $\Sigma_{\max }$ is then obtained both in heterotic and orientifold models for $\chi=\pi / 2$ and $\xi=\arcsin \sqrt{\sum_{\epsilon}\left|\hat{c}_{\epsilon}\right|^{2} /\left(2 \sum_{\epsilon}\left|\hat{c}_{\epsilon}\right|^{2}+\sum_{r}\left(c^{r}\right)^{2}\right)}$, and one finds:

$$
\Sigma_{\max }=\max _{y, z}\left\{\frac{2\left(\sum_{\epsilon}\left|\hat{c}_{\epsilon}(y, z)\right|^{2}\right)^{2}}{2 \sum_{\epsilon}\left|\hat{c}_{\epsilon}(y, z)\right|^{2}+\sum_{r}\left(c^{r}(z)\right)^{2}}\right\} .
$$

Finally, when $|c| \sim|d|$ one finds two different extrema, which generalize those seen above and compete against each other. The values of $\Sigma$ at these two extrema can be computed explicitly, although we do not report their expressions here, and $\Sigma_{\max }$ is then given by the maximum of these two extrema.

Let us next consider the case where all the parameters $d_{a b c}$ and $c_{\alpha \beta}^{a}$ are small. In such a situation one may keep only those terms in (6.14) that involve no power of $d_{a b c}$ and $c_{\alpha \beta}^{a}$. This leads to the following expression:

$$
\begin{aligned}
\Sigma(\chi, \xi, y, z) \simeq & \sin ^{4} \chi\left[\left(-\frac{2 \pm 2}{3}+\frac{2 \mp 1}{3} \zeta(y)\right) \cos ^{4} \xi-\frac{4}{3} \cos ^{2} \xi \sin ^{2} \xi\right] \\
& +\sin ^{2} \chi \cos ^{2} \chi\left[-\frac{8}{3} \kappa(x, y) \sin ^{2} \xi\right]
\end{aligned}
$$


in terms of the following functions of $x$ and $y$, which take values in the interval $[0,1]$ :

$$
\zeta(y)=\left|\delta_{a b} y^{a} y^{b}\right|^{2}, \quad \kappa(x, y)=\frac{1}{4} \delta_{a b}\left(x \bar{y}^{\bar{a}}+\bar{x} y^{a}\right)\left(x \bar{y}^{\bar{b}}+\bar{x} y^{b}\right) .
$$

In this regime, the maximization of $\Sigma$ with respect to the angles $\chi$ and $\xi$ can again be performed explicitly. For heterotic models, all the three terms are semi-negative definite. The maximum is thus obtained for $\chi=0$ and gives the value 0 . For orientifold models, the first term is instead semi-positive definite while the other two terms are as before negative-definite. The maximum is then obtained for $\chi=\pi / 2$ and $\xi=0$ and gives the value $\zeta(y)$. Since $\max _{y} \zeta(y)=1$ we then get:

$$
\Sigma_{\max } \simeq \begin{cases}0 & \text { (heterotic }), \\ 1 & \text { (orientifold }) .\end{cases}
$$

The above results for the asymptotic behavior of $\Sigma_{\max }$ can be schematically summarized in the following simple way. When $|d|,|c| \gg 1$, one can have two types of behaviors: if $|d| \gg|c|$ then $\Sigma_{\max } \sim|d|^{2}$ for heterotic models but $\Sigma_{\max } \sim 0$ for orientifold models, while if $|d| \ll|c|$ then $\Sigma_{\max } \sim 2 / 3|c|^{2}$ both for heterotic and orientifold models, and when $|d| \sim|c|$ there is a transition between these two behaviors. When $|d|,|c| \ll 1$, one finds instead the following behavior: $\Sigma_{\max } \simeq 0$ for heterotic models and $\Sigma_{\max } \simeq 1$ for orientifold models. In terms of $a$ and $b$, this implies in particular that

$$
m_{f \bar{f}}^{2} \simeq\left\{\begin{array}{l}
{\left[-4 \sin ^{4} \theta+4 \sin ^{2} \theta \cos ^{2} \theta+(1 \pm 1) a \cos ^{4} \theta\right] m_{3 / 2}^{2}, \quad a \gg 1, b \ll a,} \\
{\left[-4 \sin ^{4} \theta+4 \sin ^{2} \theta \cos ^{2} \theta+2 b \cos ^{4} \theta\right] m_{3 / 2}^{2}, \quad b \gg 1, a \ll b,} \\
{\left[-4 \sin ^{4} \theta+4 \sin ^{2} \theta \cos ^{2} \theta+\frac{3}{2}(1 \mp 1) \cos ^{4} \theta\right] m_{3 / 2}^{2}, \quad a \simeq-1, b \ll 1 .}
\end{array}\right.
$$

\section{Soft masses and flavor universality}

As a second application of the results derived in the previous sections, let us consider the condition for the flavor universality of soft supersymmetry breaking terms. This is controlled by the structure of soft scalar masses and depends on the holomorphic bisectional curvature of the scalar manifold along a given visible sector direction $v^{I}$ and the Goldstino direction $f^{I}$. More precisely, assuming again for simplicity a negligibly small cosmological constant, these masses are given by

$$
m_{v \bar{v}}^{2}=3\left(R(v, f)+\frac{1}{3}\right) m_{3 / 2}^{2}
$$

where the holomorphic bisectional curvature $R(v, f)$ is defined as

$$
R(v, f)=-R_{I \bar{J} K \bar{L}} v^{I} \bar{v}^{\bar{J}} f^{K} \bar{f}^{\bar{L}},
$$

and the vectors $v^{I}$ and $f^{I}$ are subject to the following constraints:

$$
g_{I \bar{J}} v^{I} \bar{v}^{\bar{J}}=1, \quad g_{I \bar{J}} f^{I} \bar{f}^{\bar{J}}=1, \quad g_{I \bar{J}} v^{I} \bar{f}^{\bar{J}}=0
$$


The condition of flavor universality is that $m_{v \bar{v}}^{2}$ be independent of $v^{I}$. A particularly simple and appealing first step in this direction could be to require that $m_{v \bar{v}}^{2}=0$ for every $v^{I}$, which implies $R(v, f)=-1 / 3$. In the presence of a positive cosmological constant $V$ parametrized by $\gamma=V /\left(3 m_{3 / 2}^{2}\right)$ this condition becomes $R(v, f)=-1 / 3(1+\gamma)^{-1}$. The effect of vector multiplets is instead discussed for example in $[56,57,58]$.

In the class of models that we considered, the visible sector containing the standard particles must consist of a subset of the matter fields $\Phi^{\alpha}$, while the hidden sector can involve the dilaton $S$ and a subset of the Kähler moduli and matter fields $Z^{i}=\Phi^{\alpha}, T^{A}$. We thus have $v^{S}=0, v^{A}=0, v^{\alpha} \neq 0, f^{S} \neq 0, f^{A} \neq 0$ and $f^{\alpha} \neq 0$. As before, it is convenient to introduce an angle $\theta$ and write $f^{S}=\sin \theta g^{S}$ and $f^{i}=\cos \theta h^{i}$, where now $|g|=1$ and $|h|=1$. We will again imagine that the Goldstino direction can a priori be arbitrary, as in $[11,12]$, and shall not discuss the possibilities offered by specific effects like classical fluxes or non-perturbative quantum corrections (see however [53, 54] for some recent studies on this applying to the minimal situation studied in this paper). Noticing that the bisectional curvature of the fixed coset manifold $S U(1,1) / U(1)$ describing the dilaton is trivially $R(v, g)=0$, and writing the bisectional curvature of the generic no-scale manifold $\mathcal{M}_{Y, N}$ describing the Kähler moduli and matter fields as $R(v, h)=-1 / 3+\Xi(v, h)$, one can then write $R(v, f)$ in the following form:

$$
R(v, f)=0 \cdot \sin ^{2} \theta+\left(-\frac{1}{3}+\Xi(v, h)\right) \cos ^{2} \theta .
$$

The soft scalar masses are correspondingly written as:

$$
m_{v \bar{v}}^{2}=\left[\sin ^{2} \theta+3 \Xi(v, h) \cos ^{2} \theta\right] m_{3 / 2}^{2} .
$$

The quantity $\Xi(v, h)$ can be non-zero only if the no-scale manifold $\mathcal{M}_{Y, N}$ differs from the minimal possibility $S U(1,1+n) /(U(1) \times S U(n))$. It measures the amount by which the bisectional curvature deviates from the critical value $-1 / 3$, and controls therefore the possibility of making $m_{v \bar{v}}^{2} \neq 0$ even when $\theta=0$. A quite explicit but still general expression for it can be derived by using the general properties of the geometry of noscale manifolds derived in section 2, with $Y$ homogeneous of degree three in $J^{A}$ and $N^{A}$ function of $\Phi^{\alpha} \bar{\Phi}^{\beta}$, under the simplifying assumption that the matter fields take vanishing expectation values. Using the same short-hand notation as in the previous section, in which at the considered point the moduli index $A$ is split into the values 0 corresponding to the direction parallel to $k^{A}$ and the values $a$ corresponding to the directions orthogonal to $k^{A}$, one finds:

$$
\Xi(v, h)=P_{\alpha \bar{\beta} a \bar{b}} v^{\alpha} \bar{v}^{\bar{\beta}} h^{a} \bar{h}^{\bar{b}}+Q_{\alpha \bar{\beta} \gamma \bar{\delta}} v^{\alpha} \bar{v}^{\bar{\beta}} h^{\gamma} \bar{h}^{\bar{\delta}}+\frac{1}{\sqrt{3}} \Gamma_{a \alpha \bar{\beta}} v^{\alpha} \bar{v}^{\bar{\beta}}\left(h^{0} \bar{h}^{\bar{a}}+\bar{h}^{\overline{0}} h^{a}\right),
$$

where:

$$
\begin{aligned}
P_{\alpha \bar{\beta} a \bar{b}} & =\frac{1}{3} g_{\alpha \bar{\beta}} g_{a \bar{b}}-R_{\alpha \bar{\beta} a \bar{b}} \\
Q_{\alpha \bar{\beta} \gamma \bar{\delta}} & =\frac{1}{3}\left(g_{\alpha \bar{\beta}} g_{\gamma \bar{\delta}}+g_{\alpha \bar{\delta}} g_{\gamma \bar{\beta}}\right)-R_{\alpha \bar{\beta} \gamma \bar{\delta}}
\end{aligned}
$$

The explicit form of the normalization conditions is:

$$
g_{\alpha \bar{\beta}} v^{\alpha} \bar{v}^{\bar{\beta}}=1,\left|h^{0}\right|^{2}+g_{a \bar{b}} h^{a} \bar{h}^{\bar{b}}+g_{\alpha \bar{\beta}} h^{\alpha} \bar{h}^{\bar{\beta}}=1, \quad g_{\alpha \bar{\beta}} v^{\alpha} \bar{h}^{\bar{\beta}}=0 .
$$


Moreover, invariance under the visible sector gauge symmetries clearly implies that

$$
c_{\alpha \beta}^{a} v^{\alpha} \bar{h}^{\bar{\beta}}=0 .
$$

We now want to evaluate more explicitly the quantity $\Xi$ in the specific cases of CalabiYau string models of the heterotic and orientifold types, where the Riemann tensor and the Christoffel connection are parametrized in terms of some numbers $d_{A B C}$ and $c_{\alpha \beta}^{A}$. To do so, it is again convenient to go to the canonical frame defined in sections 3 and 4 . In this way, one can use the simple characterization of the geometry derived in section 5 , and after a straightforward computation one finds that the quantities (7.7) and (7.8) reduce to the following combinations of the quantities (5.2) and (5.7):

$$
P_{\alpha \bar{\beta} a \bar{b}}=b_{\alpha \beta}^{a b}+\beta_{\alpha \beta}^{a b}-\frac{1}{2} d_{a b r} c_{\alpha \beta}^{r}, \quad Q_{\alpha \bar{\beta} \gamma \bar{\delta}}=-\left(c_{\alpha \beta}^{a} c_{\gamma \delta}^{a}+c_{\alpha \delta}^{a} c_{\gamma \beta}^{a}\right) .
$$

One then finds:

$$
\begin{aligned}
\Xi(v, h)= & \left(b_{\alpha \beta}^{a b}+\beta_{\alpha \beta}^{a b}-\frac{1}{2} d_{a b r} c_{\alpha \beta}^{r}\right) v^{\alpha} \bar{v}^{\bar{\beta}} h^{a} \bar{h}^{\bar{b}}-c_{\alpha \beta}^{a} c_{\gamma \delta}^{a} v^{\alpha} \bar{v}^{\bar{\beta}} h^{\gamma} \bar{h}^{\bar{\delta}} \\
& -\frac{1}{\sqrt{3}} c_{\alpha \beta}^{a} v^{\alpha} \bar{v}^{\bar{\beta}}\left(h^{0} \bar{h}^{\bar{a}}+\bar{h}^{\overline{0}} h^{a}\right) .
\end{aligned}
$$

We see that the structure of $\Xi(v, h)$ is absolutely identical in heterotic and orientifold models. The soft scalar masses correspondingly take the same form as derived in [21] in both types of models. We further notice that $\Xi(h)$ has a very simple dependence on $h^{0}$. This results again in two distinct behaviors for directions $h^{i}$ that are parallel and orthogonal to $k^{i}$. In the parallel direction with $h^{0}=1$ and $h^{a}, h^{\alpha}=0$, one finds a trivially vanishing $\Xi(v, h)$. In the orthogonal directions with $h^{0}=0$ and $h^{a}, h^{\alpha} \neq 0$, one instead finds a generically non-trivial $\Xi(v, h)$. Notice also that in hybrid directions where $h^{a}=0$ and $h^{0}, h^{\alpha} \neq 0$, one finds again a vanishing $\Xi(v, h)$ if the further constraints $c_{\alpha \beta}^{a} h^{\alpha} \bar{h}^{\bar{\beta}}=0$ hold true. In such a situation, the soft scalar masses would then become flavor universal:

$$
m_{v \bar{v}}^{2}=\sin ^{2} \theta m_{3 / 2}^{2} \text { if } h^{a}=c_{\alpha \beta}^{a} h^{\alpha} \bar{h}^{\bar{\beta}}=0 .
$$

\subsection{Possibility of mild sequestering}

We have just seen that one can achieve the critical value $\Xi(v, h)=0$ in a rather simple and quite generic way by requiring the Goldstino direction $h^{i}$ to be such that $h^{a}=0$ and imposing that $h^{\alpha}$ satisfies the further constraints $c_{\alpha \beta}^{a} h^{\alpha} \bar{h}^{\bar{\beta}}=0$. Such constraints always admit at least one solution, which is $h^{\alpha}=0$. This corresponds to taking $h^{i}$ parallel to $k^{i}$, which has indeed been shown to always yield $\Xi(v, h)=0$. Under favorable circumstances, there may however also exist more general solutions with $h^{\alpha} \neq 0$. Whenever they arise, these correspond to a more general choice for $h^{i}$, which also yields $\Xi(v, h)=0$ but in a potentially more flexible way. One may then try to investigate when such particular directions exist and whether it is possible to force the Goldstino direction to align along them as a result of a global symmetry, thereby realizing the idea of mild sequestering proposed in [59] (see also [60]). It was however shown in [21] that this is possible only whenever the matrices $c_{\alpha \beta}^{a}$ span a Lie algebra and $d_{a b c}$ are the symmetric symbol of this algebra. This leads to the conclusion that such a mechanism is really natural only in models where the scalar manifold is a coset, and much less natural in models where the scalar manifold is generic. 


\section{Conclusions}

In this work, we have presented a general study of the geometry of no-scale Kähler manifolds. We first derived a simple and novel general formula given by (2.24) for the curvature tensor of a completely generic no-scale Kähler manifold, in the parametrization that naturally emerges in string models with some numbers of moduli $T^{A}$ and matter fields $\Phi^{\alpha}$, as a function of the metric and the third and fourth derivatives of $e^{-K}$. This result resembles very much the expression for the curvature tensor of special Kähler manifolds in special coordinates, and displays some peculiar properties. Most importantly, we showed that at every point of such a no-scale Kähler manifold there exists a special direction along which the sectional curvature has a universal critical value. We then studied in more detail the two classes of no-scale manifolds emerging from heterotic and orientifold string models based on a generic Calabi-Yau internal manifold with a generic gauge bundle over it, characterized by some intersection numbers $d_{A B C}$ and some matrices $c_{\alpha \beta}^{A}$. We restricted for simplicity to points where only the moduli fields and not the matter fields have non-vanishing values, and introduced a canonical parametrization at such a point, where the special direction of critical curvature is aligned with one of the moduli fields $T^{0}$, while the other orthogonal directions are associated to the other moduli fields $T^{a}$ and the matter fields $\Phi^{\alpha}$. We were then able to derive two very simple and similar expressions for the Riemann tensor in these two classes of no-scale manifolds, which are given by eqs. (5.12)-(5.15) as functions of the non-trivial components $d_{a b c}$ and $c_{\alpha \beta}^{a}$ in the canonical frame defined at the reference point under consideration. We then gave a completely algebraic characterization of the conditions under which such manifolds become symmetric cosets, showing that the deviations from such a situation are essentially controlled by two combinations of parameters, called $a_{a b c d}$ and $b_{\alpha \beta}^{a b}$ and defined by (5.1) and (5.2). This allowed us to argue that while in the case of one modulus field and any number of matter fields one unavoidably gets a maximally symmetric manifold, and in the case of two moduli fields and zero matter fields one finds a disconnected one-parameter family of models separated by a unique possible coset manifold, in all other cases one obtains a connected multi-parameter family of models where possible coset manifolds represent isolated points. We then observed that the no-scale manifolds arising in heterotic and orientifold models display a kind of duality, in the sense that the associated Riemann tensors differ only by the sign of the contribution depending on $a_{a b c d}$, while all the remaining terms and in particular those depending on $b_{\alpha \beta}^{a b}$ have the same sign. As a result, the heterotic and orientifold no-scale manifolds coincide if $a_{a b c d}$ vanishes, while $b_{\alpha \beta}^{a b}$ may still be arbitrary, so that one may or may not get a coset manifold.

As an application of the results that we derived for the geometry of no-scale Kähler manifolds, we studied the general structure of those scalar masses that are entirely controlled by supersymmetry breaking splitting effects, in string models where the universal dilaton sector and a generic no-scale sector involving an arbitrary number of Kähler moduli and matter fields are included. We used for this the general form that the Kähler potential must take in such a situation and assumed that a completely generic superpotential may arise and trigger supersymmetry breaking in way involving all the above fields. As a first

application, we studied the average sGoldstino square mass $m_{f \bar{f}}^{2}$ in the hidden sector of superfields taking non-vanishing expectation values, defined by the Goldstino direction $f^{i}$ 
of supersymmetry breaking. This direction has components $f^{S}$ and $f^{A}, f^{\alpha}$ in the dilaton and no-scale sectors, with a relative magnitude that is weighted by an angle $\theta$. We derived an explicit expression for $m_{f \bar{f}}^{2}$ in the canonical frame, given by eqs. (6.5) and (6.14), and showed that it is essentially controlled by the quantities $a_{a b c d}$ and $b_{\alpha \beta}^{a b}$ that parametrize the deviations of the geometry from a coset situation. More precisely, what matter are the extremal values $( \pm a)_{\text {up }}$ and $b_{\text {up }}$ that can be achieved for their contractions $\pm a(\tilde{y})$ and $b(\tilde{y}, \tilde{z})$ along real normalized directions $\tilde{y}^{a}$ and $\tilde{z}^{\alpha}$ in the subspaces of the non-minimal moduli $T^{a}$ and the matter fields $\Phi^{\alpha}$, the two signs applying respectively to heterotic and orientifold models. We first showed that a qualitative necessary condition for being able to achieve even for vanishing $\theta$ a positive $m_{f \bar{f}}^{2}$, which is necessary and sufficient for the existence of a metastable supersymmetry breaking vacuum if one allows the superpotential to be tuned, is that at least one of the two quantities $( \pm a)_{\text {up }}$ and $b_{\text {up }}$ be positive. We then also derived an upper bound on the absolute magnitude of $m_{f \bar{f}}^{2}$, which also represents an upper bound on the mass of the lightest particle in the hidden sector, given by (6.31). In particular, this formula shows that when the effects of moduli fields dominate one finds at best $m_{f \bar{f}}^{2} \simeq 2 a m_{3 / 2}^{2}$ for $a \gg 1$ in heterotic models and $m_{f \bar{f}}^{2} \simeq 16 / 5 m_{3 / 2}^{2}$ when $a \simeq-1$ in orientifold models, while when the effects of matter fields dominate one can achieve $m_{f \bar{f}}^{2} \simeq 2 b m_{3 / 2}^{2}$ for $b \gg 1$ in both models. We finally argued that $m_{f \bar{f}}^{2}$ can generically be made hierarchically larger than $m_{3 / 2}^{2}$ by suitable choosing the vacuum point to make $a$ or $b$ large. More precisely, with a single modulus and any number of matter fields $m_{f \bar{f}}^{2}$ is bounded, and with any number of moduli and zero matter fields it can be arbitrarily large in heterotic models and is bounded in orientifold models, but in any other situation one can get an arbitrarily large result both for heterotic and orientifold models, except for the isolated cases corresponding to coset manifolds. As a second application we studied the soft scalar square masses $m_{v \bar{v}}^{2}$ in the visible sector of superfields taking vanishing expectation values, defined by an arbitrary direction $v^{\alpha}$ in flavor space. We presented a simple general expression for $m_{v \bar{v}}^{2}$ in the canonical frame, given by eqs. (7.5) and (7.12), and emphasized that it is identical in form for heterotic and orientifold models. We first investigated the conditions under which $m_{v \bar{v}}^{2}$ can be flavor universal, as required by phenomenological considerations. We then showed that $m_{v \bar{v}}^{2}$ may be forced to be flavor universal by suitably orienting the Goldstino direction and that this mild sequestering mechanism may be implemented by postulating the existence of some approximate global symmetries in the hidden sector.

To conclude, let us remark that the general and model-independent results we derived for $m_{f \bar{f}}^{2}$ and $m_{v \bar{v}}^{2}$ also display interesting correlations. Most importantly, we see that when the Goldstino direction satisfies the constraints $f^{a}=c_{\alpha \beta}^{a} f^{\alpha} \bar{f}^{\bar{\beta}}=0$, one interestingly finds

that $m_{f \bar{f}}^{2}$ is given by the bounded and sign-indefinite result (6.16) and $m_{v \bar{v}}^{2}$ is given by the flavor-universal and positive result (7.13). This seems to suggest that there is no cheap way of simultaneously achieving a large $m_{f \bar{f}}^{2}$ and a flavor universal $m_{v \bar{v}}^{2}$.

\section{Acknowledgements}

This work was supported by the Swiss National Science Foundation. We thank M. Trigiante for useful discussions and K. Sinha for interesting correspondence. 


\section{References}

[1] E. Witten, Dimensional reduction of superstring models, Phys. Lett. B 155 (1985) 151.

[2] S. Gukov, C. Vafa and E. Witten, CFT's from Calabi-Yau four-folds, Nucl. Phys. B 584 (2000) 69 [Erratum-ibid. B 608 (2001) 477] [hep-th/9906070].

[3] S. B. Giddings, S. Kachru and J. Polchinski, Phys. Rev. D 66 (2002) 106006 [hep-th/0105097].

[4] S. Kachru, R. Kallosh, A. Linde and S. P. Trivedi, De Sitter vacua in string theory, Phys. Rev. D 68 (2003) 046005 [hep-th/0301240].

[5] M. Grana, Flux compactifications in string theory: a comprehensive review, Phys. Rept. 423, 91 (2006) [hep-th/0509003].

[6] J. P. Derendinger, L. E. Ibanez and H. P. Nilles, On the Low-Energy $d=4, N=1$ Supergravity Theory Extracted from the $d=10, N=1$ Superstring, Phys. Lett. B 155 (1985) 65.

[7] M. Dine, R. Rohm, N. Seiberg and E. Witten, Gluino condensation in superstring models, Phys. Lett. B 156 (1985) 55.

[8] S. Ferrara, C. Kounnas and M. Porrati, General dimensional reduction of ten-dimensional supergravity and superstring, Phys. Lett. B 181 (1986) 263.

[9] M. Gomez-Reino and C. A. Scrucca, Locally stable non-supersymmetric Minkowski vacua in supergravity, JHEP 0605 (2006) 015 [hep-th/0602246].

[10] F. Denef and M. R. Douglas, Distributions of nonsupersymmetric flux vacua, JHEP 0503 (2005) 061 [arXiv:hep-th/0411183].

[11] V. S. Kaplunovsky and J. Louis, Model independent analysis of soft terms in effective supergravity and in string theory, Phys. Lett. B 306 (1993) 269 [hep-th/9303040].

[12] A. Brignole, L. E. Ibanez and C. Munoz, Towards a theory of soft terms for the supersymmetric Standard Model, Nucl. Phys. B 422 (1994) 125 [Errat. B 436 (1995) 747] [hep-ph/9308271].

[13] H. B. Kim and C. Munoz, An Analysis of soft terms in Calabi-Yau compactifications, Z. Phys. C 75 (1997) 367 [hep-ph/9608214].

[14] E. Cremmer, S. Ferrara, C. Kounnas and D. V. Nanopoulos, Naturally vanishing cosmological constant in N=1 supergravity, Phys. Lett. B 133 (1983) 61.

[15] J. R. Ellis, A. B. Lahanas, D. V. Nanopoulos and K. Tamvakis, No-Scale supersymmetric standard model, Phys. Lett. B 134 (1984) 429.

[16] R. Barbieri, E. Cremmer and S. Ferrara, Flat and positive potentials in N=1 supergravity, Phys. Lett. B 163 (1985) 143.

[17] M. Gomez-Reino and C. A. Scrucca, Constraints for the existence of flat and stable nonsupersymmetric vacua in supergravity, JHEP 0609 (2006) 008 [hep-th/0606273].

[18] S. P. Li, R. B. Peschanski and C. A. Savoy, Mass degeneracy and the superhiggs mechanism, Nucl. Phys. B 289 (1987) 206.

[19] C. Andrey and C. A. Scrucca, Mildly sequestered supergravity models and their realization in string theory, Nucl. Phys. B 834 (2010) 363 [arXiv:1002.3764].

[20] L. Covi, M. Gomez-Reino, C. Gross, J. Louis, G. A. Palma and C. A. Scrucca, De Sitter vacua in no-scale supergravities and Calabi-Yau string models, JHEP 0806 (2008) 057 [arXiv:0804.1073].

[21] C. Andrey and C. A. Scrucca, Sequestering by global symmetries in Calabi-Yau string models, Nucl. Phys. B 851 (2011) 245 [arXiv:1104.4061]. 
[22] D. Lust, S. Reffert, W. Schulgin and S. Stieberger, Moduli stabilization in type IIB orientifolds. I: Orbifold limits, Nucl. Phys. B 766 (2007) 68 [hep-th/0506090].

[23] D. Lust, S. Reffert, E. Scheidegger, W. Schulgin and S. Stieberger, Moduli stabilization in type IIB orientifolds. II, Nucl. Phys. B 766 (2007) 178 [hep-th/0609013].

[24] K. Choi, A. Falkowski, H. P. Nilles and M. Olechowski, Soft supersymmetry breaking in KKLT flux compactification, Nucl. Phys. B 718 (2005) 113 [hep-th/0503216].

[25] E. Calabi and E. Vesentini, On compact, locally symmetric Kähler manifolds, Ann. Math. 71 (1960) 3 .

[26] P. Candelas, G. T. Horowitz, A. Strominger and E. Witten, Vacuum configurations for superstrings, Nucl. Phys. B 258 (1985) 46.

[27] E. Witten, New issues in manifolds of SU(3) holonomy, Nucl. Phys. B 268 (1986) 79.

[28] J. Distler and B. R. Greene, Aspects of (2,0) string compactifications, Nucl. Phys. B 304, 1 (1988).

[29] S. Cecotti, S. Ferrara and L. Girardello, A topological formula for the Kähler potential of 4-D $N=1, N=2$ strings and its implications for the moduli problem, Phys. Lett. B 213 (1988) 443.

[30] P. Candelas and X. de la Ossa, Moduli space of Calabi-Yau manifolds, Nucl. Phys. B 355 (1991) 455.

[31] L. J. Dixon, V. Kaplunovsky and J. Louis, On effective field theories describing (2,2) vacua of the heterotic string, Nucl. Phys. B 329 (1990) 27.

[32] F. Paccetti Correia and M. G. Schmidt, Moduli stabilization in heterotic M-theory, Nucl. Phys. B 797 (2008) 243 [arXiv:0708.3805].

[33] S. Gurrieri, A. Lukas and A. Micu, Heterotic string compactifications on half-flat manifolds II, JHEP 0712 (2007) 081 [arXiv:0709.1932].

[34] I. Benmachiche, J. Louis and D. Martinez-Pedrera, The effective action of the heterotic string compactified on manifolds with SU(3) structure, Class. Quant. Grav. 25 (2008) 135006 [arXiv:0802.0410].

[35] E. I. Buchbinder and B. A. Ovrut, Vacuum stability in heterotic M-theory, Phys. Rev. D 69 (2004) 086010 [hep-th/0310112].

[36] M. Gunaydin, G. Sierra and P. K. Townsend, The geometry of N=2 Maxwell-Einstein supergravity and Jordan algebras, Nucl. Phys. B 242 (1984) 244.

[37] E. Cremmer, C. Kounnas, A. Van Proeyen, J. P. Derendinger, S. Ferrara, B. de Wit and L. Girardello, Vector multiplets coupled to N=2 supergravity: superhiggs effect, flat potentials and geometric structure, Nucl. Phys. B 250 (1985) 385.

[38] J. Louis, Generalized Calabi-Yau compactifications with D-branes and fluxes, Fortsch. Phys. 53 (2005) 770.

[39] R. Blumenhagen, B. Kors, D. Lust and S. Stieberger, Four-dimensional string compactifications with D-Branes, orientifolds and fluxes, Phys. Rept. 445 (2007) 1 [hep-th/0610327].

[40] T. W. Grimm and J. Louis, The Effective action of $N=1$ Calabi-Yau orientifolds, Nucl. Phys. B 699 (2004) 387 [hep-th/0403067].

[41] M. Grana, T. W. Grimm, H. Jockers and J. Louis, Soft supersymmetry breaking in Calabi-Yau orientifolds with D-branes and fluxes, Nucl. Phys. B 690 (2004) 21 [hep-th/0312232].

[42] H. Jockers and J. Louis, The effective action of D7-branes in $N=1$ Calabi-Yau orientifolds, Nucl. Phys. B 705 (2005) 167 [hep-th/0409098]. 
[43] R. D'Auria, S. Ferrara and M. Trigiante, C-map, very special quaternionic geometry and dual Kahler spaces Phys. Lett. B 587 (2004) 138 [hep-th/0401161].

[44] R. D'Auria, S. Ferrara and M. Trigiante, Homogeneous special manifolds, orientifolds and solvable coordinates, Nucl. Phys. B 693 (2004) 261 [hep-th/0403204].

[45] E. Cremmer and A. Van Proeyen, Classification of Kahler manifolds in N=2 vector multiplet supergravity couplings, Class. Quant. Grav. 2 (1985) 445.

[46] L. Covi, M. Gomez-Reino, C. Gross, J. Louis, G. A. Palma and C. A. Scrucca, Constraints on modular inflation in supergravity and string theory, JHEP 0808, 055 (2008) [arXiv:0805.3290].

[47] M. Gómez-Reino and C. A. Scrucca, Metastable supergravity vacua with $F$ and D supersymmetry breaking, JHEP 0708 (2007) 091 [arXiv:0706.2785].

[48] L. Brizi and C. A. Scrucca, The lightest scalar in theories with broken supersymmetry, JHEP 1111 (2011) 013 [arXiv:1107.1596].

[49] P. G. Camara, L. E. Ibanez and A. M. Uranga, Flux induced SUSY breaking soft terms, Nucl. Phys. B 689 (2004) 195 [hep-th/0311241].

[50] D. Lust, S. Reffert and S. Stieberger, Flux-induced soft supersymmetry breaking in chiral type IIB orientifolds with D3 / D7-branes, Nucl. Phys. B 706 (2005) 3 [hep-th/0406092].

[51] P. G. Camara, L. E. Ibanez and A. M. Uranga, Flux-induced SUSY-breaking soft terms on D7-D3 brane systems, Nucl. Phys. B 708 (2005) 268 [hep-th/0408036].

[52] D. Lust, S. Reffert and S. Stieberger, MSSM with soft SUSY breaking terms from DY-branes with fluxes, Nucl. Phys. B 727 (2005) 264 [hep-th/0410074].

[53] L. Covi, M. Gomez-Reino, C. Gross, G. A. Palma and C. A. Scrucca, Constructing de Sitter vacua in no-scale string models without uplifting, JHEP 0903 (2009) 146 [arXiv:0812.3864].

[54] B. de Carlos, A. Guarino and J. M. Moreno, Flux moduli stabilisation, supergravity algebras and no-go theorems, JHEP 1001 (2010) 012 [arXiv:0907.5580].

[55] B. S. Acharya, G. Kane and E. Kuflik, String theories with moduli stabilization imply nonthermal cosmological history, and particular dark matter [arXiv:1006.3272].

[56] S. Ferrara, C. Kounnas and F. Zwirner, Mass formulae and natural hierarchy in string effective supergravities, Nucl. Phys. B 429 (1994) 589, Errat. B 433 (1995) 255 [hep-th/9405188].

[57] E. Dudas and S. K. Vempati, Large D-terms, hierarchical soft spectra and moduli stabilisation, Nucl. Phys. B 727 (2005) 139 [hep-th/0506172].

[58] C. A. Scrucca, Soft masses in superstring models with anomalous U(1) symmetries, JHEP 0712 (2007) 092 [arXiv:0710.5105].

[59] S. Kachru, L. McAllister and R. Sundrum, Sequestering in string theory, JHEP 0710 (2007) 013 [hep-th/0703105].

[60] M. Schmaltz and R. Sundrum, Conformal sequestering simplified, JHEP 0611 (2006) 011 [hep-th/0608051]. 